\title{
Power production assessment for wave energy converters: Overcoming the perils of the power matrix
}

Proc IMechE Part M:

J Engineering for the Maritime Environment 2018, Vol. 232(I) 50-70 (c) IMechE 2017

Reprints and permissions: sagepub.co.uk/journalsPermissions.nav DOI: |0.1|77/|47509021773|67| journals.sagepub.com/home/pim (SSAGE

\author{
Alexis Mérigaud and John V Ringwood
}

\begin{abstract}
Wave energy converter power production assessment, usually carried out using a power matrix, is essential for the appraisal of new wave energy converter technologies and for the planning of specific wave energy projects. Errors in power assessment may arise, both from an inaccurate description of the wave energy converter dynamics and from an excessively simplified representation of wave spectra in the power matrix approach. Ideally, the wave energy converter output should be computed in every individual sea state of the wave dataset considered, without the assumption of any parametric spectral shape. However, computationally efficient methods are necessary to achieve such extensive wave energy converter simulation. The non-linear frequency-domain technique is significantly faster than Runge-Kutta timedomain simulations, without affecting the representation of radiation forces and non-linear dynamics. In this article, the two main sources of errors in wave energy converter power assessment, namely the power matrix representation and wave energy converter modelling inaccuracies, are jointly studied and put into perspective, using four case studies (two wave energy converter systems in two locations). It is found that both types of errors can be of comparable magnitude. The non-linear frequency-domain technique simulation technique is shown to be a computationally efficient tool, retaining a realistic representation of the device dynamics while avoiding the use of a power matrix, thus preserving accurate representation of both the sea states and the wave energy converter, at little computational expense. Aside from those main results, the issue of the length and number of simulations, necessary to achieve average power estimates with sufficient accuracy in every sea state, is addressed in detail.
\end{abstract}

\section{Keywords}

Power assessment, wave spectrum, numerical simulation, wave energy converter, average power estimates, power matrix, non-linear models, non-linear frequency domain

Date received: 12 March 2017; accepted: 7 August 2017

\section{Introduction}

At the current development stage of wave energy converter (WEC) technologies, WEC power production assessment is essential to evaluate and compare the potential of various wave energy conversion technologies at different locations. ${ }^{1-8}$ In the future, power assessment will also be instrumental in determining the economic viability of specific wave energy projects. In a wider sense, WEC power production assessment covers studies concerning the variability of WEC production over different time, and geographical, scales. ${ }^{7,9-12}$

The general methodology for power production assessment consists of using historical wave data for a specific location, over typically a year or more, and simulating the WEC power output over the chosen duration. The findings of Torsethaugen ${ }^{13}$ suggest that a
1 -year period is insufficient and that a 10 -year assessment is necessary. At every time step of the period considered, that is, typically every $1-3 \mathrm{~h}$, the power output is computed from the knowledge of the wave spectrum - or the wave elevation data if those are available at the location of interest. As a consequence, power assessment involves the computation of the WEC output in a large number of sea states, which is

Centre for Ocean Energy Research, Department of Electronic Engineering, Maynooth University, Maynooth, Ireland

\section{Corresponding author:}

Alexis Mérigaud, Centre for Ocean Energy Research, Department of Electronic Engineering, Maynooth University, Maynooth, Co. Kildare, Ireland.

Email: alexis.merigaud.2015@mumail.ie 
carried out, in the vast majority of studies, using the power matrix (PM) representation of the device.

The PM is a concise WEC representation, showing the WEC response, in terms of average power production, as a function of two parameters describing the sea state. Thus, in a PM, sea states are characterised by means of only two parameters, generally the significant wave height $H_{m_{0}}$ and the peak wave period $T_{p}$, assuming a parametric spectral shape such as a Joint North Sea Wave Project (JONSWAP) ${ }^{14}$ or Bretschneider ${ }^{15}$ spectrum for the waves. The device output is computed over a range of $H_{m_{0}}, T_{p}$ pairs, each pair representing a cell of the PM. Then, in a given sea state, the power output is predicted by matching the $H_{m_{0}}, T_{p}$ parameters of the spectrum with the corresponding cell of the PM, using interpolation between neighbouring PM cells, as necessary.

Furthermore, if only the annual power value is of interest, the PM can simply be multiplied by the scatter plot of the site considered, ${ }^{1}$ the value in each cell of the scatter plot representing the probability of occurrence of a given $H_{m_{0}}, T_{p}$ pair. The PM approach therefore has appealing computational properties, compared to the performance of a WEC simulation for every individual sea state.

The benefits of the PM are even more obvious when the WEC dynamical description includes non-linear effects, such as quadratic viscous drag terms, non-linear power take-off (PTO) forces, a non-linear FroudeKrylov force description or non-linear mooring effects. In such cases, for a given spectrum, time-domain simulations are the standard method of obtaining the WEC output. ${ }^{16}$ The computational cost of time-domain simulations is significant, in comparison to linear frequencydomain WEC output calculations, which simply necessitate a multiplication of the input wave spectrum by the WEC linear transfer function. Therefore, while computing the WEC output by means of time-domain simulations, for all individual spectra of the period considered, would represent a significant computational burden, filling in the cells of a PM and using the $\left(H_{m_{0}}, T_{p}\right)$ scatter diagram is much less demanding.

However, regardless of the linearity of the WEC description, the PM approach can be subject to significant limitations in terms of accuracy, since twoparameter wave spectra cannot reflect the variety of spectral shapes which can be encountered in real seas. In particular, bi- or multi-modal sea states are poorly represented with just two parameters. Given that the more energetic sea states are closer to standard, theoretical spectral shapes, it could be assumed that the error committed when using a PM is relatively modest. ${ }^{17}$ However, recent work ${ }^{2}$ tends to show that, depending on the WEC type and location, using a PM approach, and assuming a JONSWAP spectral shape, can result in an error of up to $20 \%$ in terms of average annual energy production, compared to computing the WEC output using the actual spectra. On a sea state by

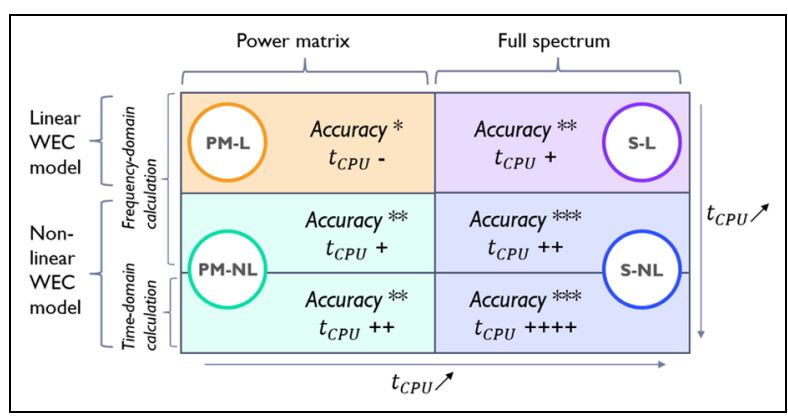

Figure I. Four main categories of WEC representations for power assessment, along with the associated accuracy and computational cost.

sea-state basis (i.e. for a given 3-h spectrum), the error committed can amount to $200 \%$.

In summary, there are two potential sources of error in typical power production assessment computations:

- The dynamical description of the device can be insufficiently realistic. In particular, linear frequency-domain models, although computationally efficient, fail in capturing non-linear dynamics, especially under controlled conditions. ${ }^{18}$ Timedomain models can be used in order to include non-linear effects; however, time-domain simulation over the entire set of sea states for an extended period is computationally demanding. Therefore, time-domain models are normally used to fill in each cell of a PM, ${ }^{1,5}$ rather than to simulate the WEC in every individual sea state.

- The sea-state description, through the two parameters of a PM, can also be the cause of incorrect estimates.

Finally, within the scope of power production assessment studies, four main types of WEC representations can be identified and are shown in Figure 1:

1. A PM, with the entries determined from a linear WEC model, hereafter referred to as PM-L;

2. A PM, with the entries determined from a nonlinear WEC model, or determined through experimental measurements (PM-NL); ${ }^{1,5}$

3. A linear WEC representation, simulated in every individual sea state without assuming any predefined spectral shape (S-L), such as in De Andrés et al. $^{2}$ for a Portuguese location;

4. A non-linear WEC representation, simulated in every individual sea state without assuming any pre-defined spectral shape (S-NL).

PM-L requires negligible computational effort, but accumulates the two sources of error described above, that is, in the description of sea states and WEC dynamics. 
PM-NL, although describing the system dynamics accurately, is subject to errors related to the misrepresentation of the sea spectra. Since only the PM cells have to be filled in, the computational cost of PM-NL is moderate, even if time-domain simulations are used, such as in Babarit et al. ${ }^{1}$ and Rusu and Onea. ${ }^{5}$

$\mathrm{S}-\mathrm{L}$, in contrast, represents the wave spectra accurately, at the expense of the description of non-linear WEC dynamics. The computational cost associated with S-L is small, since linear power computation for each individual spectrum is extremely fast.

There exists a 'grey zone' between PMs and full spectrum representation. For example, it has been suggested to add more spectral parameters to the PM entries. Such additional spectral parameters can characterise the whole spectral shape, such as wave groupiness and spectral bandwidth. ${ }^{19}$ Describing individual wave systems which compose the spectrum could also be considered. $^{20}$ However, a multi-dimensional PM involves a large number of cells to be filled in; for example, with four parameters, assuming that the range of values for each parameter is discretised into 10 intervals, the number of bins to fill in is in the order of 10,000 .

Another interesting method is the MaxDiss selection technique, used in De Andrés et al.: ${ }^{2}$ the PM has three entries, namely $H_{m_{0}}, T_{p}$ and a broadness parameter $\epsilon_{0}$. Within the historical wave data at a specific location, a limited number of spectra are chosen, which span the range of $\left(H_{m_{0}}, T_{p}, \epsilon_{0}\right)$ triplets encountered at the site under study. Then, instead of assuming a JONSWAP spectral shape, the PM is built using the selected sea states, combined with an interpolation technique. The resulting PM is then based on a more faithful representation of the spectra which characterise the location, at moderate computational cost. Although the WEC models studied in De Andrés et al. $^{2}$ are linear, the technique could be usefully applied to non-linear WEC descriptions. However, regardless of the WEC dynamics, the resulting WEC representation is site-specific, and the procedure has to be carried out again for studies in other locations.

S-NL should represent the ideal WEC power assessment method, in terms of accuracy, since both the sea state and the device are accurately represented, without any approximation other than the limitations inherent to any physical WEC model. However, it is generally considered that non-linearities in the WEC model have to be handled by means of time-domain simulations (bottom right cell in Figure 1), which makes S-NL computation over the whole dataset relatively intractable.

Nevertheless, other calculation methods exist, which allow for computationally efficient estimation of the WEC outputs while taking into account the system non-linearities. In particular, the recently proposed non-linear frequency-domain (NLFD) method $^{21,22}$ enables the non-linear, steady-state system response to a periodic, polychromatic wave signal, to be efficiently and accurately computed. NLFD relies on the projection of the dynamical equations onto a basis of harmonic sinusoids. The resulting set of non-linear equations is then solved using a gradient-based algorithm. NLFD can be successfully applied to WEC simulation, ${ }^{22}$ since:

- Without requiring any approximation of the radiation force, the computational gain is at least two orders of magnitude lower than a typical secondorder Runge-Kutta (RK2) integration (using a direct computation of the radiation force convolution product).

- Due to the quadratic convergence rate of the gradient-based algorithm, the estimated WEC trajectory can be made arbitrarily accurate at little or no additional computational cost. The case studies of Mérigaud and Ringwood ${ }^{22}$ show that timedomain results converge to NLFD results, when the time-domain integration step tends to zero.

When such computationally efficient calculation methods are available, the benefits of using a PM-NL are no longer obvious, with respect to a computation of the WEC output for all the individual spectra, using a frequency-domain formalism to solve for the non-linear WEC motion (last column, middle row in Figure 1).

This article puts into perspective the two main sources of inaccuracies in WEC power assessment: those related to the WEC dynamical description, and those due to the misrepresentation of the sea state in the PM approach. Furthermore, it is shown that both sources of error can be overcome simultaneously, by computing the non-linear WEC output in all individual sea states (S-NL) using the NLFD calculation method.

Two reactively controlled WEC systems, namely a flap-type WEC and a spherical heaving point absorber, are studied in two diverse locations off the Irish coast. For each WEC system, two models are built: a linearised model, and a non-linear model which includes device-specific non-linear forces. Then, for each WEC system and each location, the annual power output can be computed in four different ways: by filling-in a PM, using either the linear WEC model (PM-L) or the nonlinear WEC model (PM-NL), or by computing the WEC output in each individual spectrum, again using either the linear WEC model (S-L) or the non-linear WEC model (S-NL). All non-linear computations (in PM-NL and S-NL) are carried out at a modest computational expense, using NLFD. In particular, S-NL is shown to be achievable at reasonable computational cost, while being able to capture both the non-linear device dynamics and the complexity of real ocean wave spectral shapes.

Finally, although the wave elevation data itself were available for this specific study, the power assessment methodologies considered are all based on a spectral description of the sea states. Indeed, except for the strongest storms and for shallow water, the Gaussian model of ocean waves is accurate, ${ }^{23}$ and can then realistically describe the wave excitation when WECs are in 
operation. Furthermore, long enough wave elevation records are only available at specific locations where measurement buoys have been operating consistently for several years. In contrast, spectra can not only be measured but also reconstructed in areas of interest by means of advanced meteorological models, such as in Gallagher et al. ${ }^{24}$ Therefore, power assessment methods based on a spectral description of ocean waves are more widely applicable.

The rest of this article is divided into five sections: The section entitled NLFD simulation introduces the NLFD technique, which is the core of the proposed power assessment methodology. Then, WEC models, supported by material in Appendix 1, presents the two WEC systems, with their linear and non-linear variants. Wave dataset describes the two wave datasets considered in the study, and section 'Number and length of simulations per sea state' addresses the important issue of the number of simulations to carry out for each sea state. Power assessment results, using the four types of device representation (PM-L, PM-NL, S-L and S-NL), are shown and compared in section 'Numerical results', for the two WEC systems and the two locations. Finally, the main outcomes of the study are discussed in the sections 'Discussion' and 'Conclusion'.

\section{NLFD simulation}

\section{NLFD representation of the dynamical equations}

The NLFD simulation method underpins all the nonlinear, numerical investigations presented in this article. It constitutes a useful way to enhance the computational efficiency in the context of extensive WEC power calculations with non-linear dynamical models.

Let us consider a WEC with 1 degree of freedom (DoF) $x$. A generic formulation for the dynamical equation is

$$
g_{l}(x, \dot{x}, \ddot{x})-f_{n l}(x, \dot{x}, t)+f_{e}(t)=0
$$

where

- $g_{l}$ includes the inertial terms (the presence of inertial terms in $g_{l}$ is the reason why the notation $g$ is used instead of $f$ ), and the linearly modelled forces;

- $f_{n l}$ denotes forces which are modelled in a nonlinear way;

- $f_{e}$ denotes additive excitation forces (possibly obtained from linear wave theory).

The formulation of equation (1) applies well to nonlinear extensions of the well-known Cummins equation. ${ }^{25}$ Although they are not theoretically consistent, such descriptions allow for including the most significant non-linear effects, such as viscous drag, mooring line forces, non-linear Froude-Krylov forces and nonlinear PTO forces, while avoiding the computational burden of computational fluid dynamics (CFD) and the financial cost of physical experiments. ${ }^{16}$

Typically, if hydrodynamic radiation and hydrostatic restoring forces are linearly modelled, $g_{l}$ can be expressed as

$$
g_{l}(x, \dot{x}, \ddot{x})=\left(\mu+\mu_{\infty}\right) \ddot{x}+\int_{-\infty}^{t} k_{\text {rad }}(t-\tau) \dot{x}(\tau) d \tau+k_{h} x
$$

where

- $\mu$ is the WEC inertia;

- $k_{h}$ is the hydrostatic stiffness coefficient;

- The radiation forces are computed as the sum of an inertial term $\mu_{\infty} \ddot{\zeta}$ and a convolution product between the past values of the velocity and the radiation impulse response function $k_{\text {rad }}$.

If some terms of $g_{l}$, for example, the restoring force, are to be modelled non-linearly, they have to be moved from $g_{l}$ to $f_{n l}$.

Let us consider a periodic incoming wave or excitation force signal, $\eta(t)$ or $f_{e}(t)$, which can be described as a weighted sum of harmonic sinusoids, such as

$$
\eta(t) \approx \sum_{k=1}^{N} a_{\eta_{k}} \cos \left(\omega_{k} t\right)+b_{\eta_{k}} \sin \left(\omega_{k} t\right)
$$

where $\forall k \in \llbracket 1 ; N \rrbracket, \omega_{k}=k \omega_{0}, \omega_{0}=2 \pi / T, T$ is the time period after which $\eta$ is repeated, and the component amplitudes $a_{k}$ and $b_{k}$ are derived from the wave spectrum.

It is assumed that the motion of the WEC, subject to the wave signal $\eta$, has a periodic steady-state solution, which can also be described through a projection onto the same trigonometric basis so that

$$
x(t) \approx \mu_{x}+\sum_{k=1}^{N} a_{x k} \cos \left(\omega_{k} t\right)+b_{x k} \sin \left(\omega_{k} t\right)
$$

This way, the dynamical equation (1) can be projected onto the basis of trigonometric polynomials, resulting in a non-linear vector equation

$$
M X-F(X)-F_{e}=0
$$

where

- $G_{l}(X)=M X$ is the matrix transcription of the linear terms of $g_{l}$. In particular, the linear timedomain radiation terms simplify into the frequencydependent radiation added mass and damping $A_{\text {rad }}(\omega)$ and $B_{\text {rad }}(\omega)$. For example, when both radiation and hydrostatic restoring forces are linearly modelled, $\forall i, j \in \llbracket 1, \ldots, N \rrbracket^{2}$ 


$$
\begin{aligned}
& M_{i j}= \begin{cases}-\omega_{i}^{2}\left(\mu+A_{\text {rad }}\left(\omega_{i}\right)\right)+k_{h}, & i=j \\
0, & i \neq j\end{cases} \\
& M_{i+N, j+N}=M_{i, j} \\
& M_{i, j+N}= \begin{cases}\omega_{i} B_{\text {rad }}\left(\omega_{i}\right), & i=j \\
0, & i \neq j\end{cases} \\
& M_{i+N, j}=-M_{i, j+N} \\
& \text { and } M_{2 N+1,2 N+1}=k_{h}
\end{aligned}
$$

- $F(X)$ is the projection, similar to equation (3), of the non-linear forces $f_{n l}$ onto the harmonic basis so that $\forall i \in \llbracket 1, \ldots, N \rrbracket$

$$
\begin{aligned}
& F_{i}(X)=\frac{2}{T} \int_{0}^{T} f_{n l}\left(x_{X}, \dot{x}_{X}, t\right) \cos \left(\omega_{i} t\right) d t \\
& F_{i+N}(X)=\frac{2}{T} \int_{0}^{T} f_{n l}\left(x_{X}, \dot{x}_{X}, t\right) \sin \left(\omega_{i} t\right) d t
\end{aligned}
$$

where the subscript $X$ indicates that the time-domain periodic trajectory $x$ depends on the set of coefficients contained in $X$;

- $F_{e}$ represents the projection of additive force terms. For example, $F_{e}$ can correspond to the linear excitation forces resulting from the wave signal $\eta$.

\section{Solution method}

Defining $H(X):=M X-F(X)-F_{e}$, equation (5) is a non-linear vector equation which can be expressed as

$$
H(X)=0
$$

The Jacobian of $H$ can be computed, which makes it possible to use Newton's algorithm to solve for $H(X)=0$. The Newton procedure is iterated until $\|H(X)\|^{2}$ is smaller than some given threshold $\nu$. The computation of the Jacobian of the non-linear term, $F(X)$, at each iteration, necessitates $2 N$ fast Fourier transforms (FFTs). Details of the solution method and the gradient computation can be found in Spanos et al. ${ }^{21}$ Further discussion about the NLFD method, in particular in terms of a comparison with time-domain results and computational performance, can be found in Mérigaud and Ringwood. ${ }^{22}$

As already stressed in the section 'Introduction', it has to be noted that NLFD is not an approximation of the results obtained with time-domain integration. On the contrary, results presented in Mérigaud and Ringwood $^{22}$ tend to show that time-domain results obtained from RK2 integration get closer and closer to NLFD results when the integration step goes to zero.

Finally, it is important to keep in mind that the calculation of the non-linear forces equation (7) and their gradient, at each iteration of Newton's method, implies that the non-linear forces can be expressed as analytical and differentiable functions of $x$.

\section{WEC models}

Here, two generic WEC systems are considered: a flaptype oscillating wave-surge converter and a spherical heaving point absorber. Two mathematical model types are used to describe each system: a linear one, and a non-linear one, which includes more realistic dynamical effects.

The difference between results of the linear and nonlinear models represents the error encountered when excessively simplifying the WEC description.

\section{Flap-type WEC}

The first device considered is a flap-type WEC, for which the radiation, hydrostatic restoring force and excitation forces are modelled linearly. The flap is subject to a quadratic viscous drag term with moment

$$
f_{v}(\dot{x})=-C_{v} \dot{x}|\dot{x}|
$$

where $x$ denotes the angular displacement of the flap, and $C_{v}$ is given, as in Bacelli, ${ }^{26}$ by

$$
C_{v}=\frac{1}{8} \rho C_{d} L H^{4}
$$

where $H$ and $L$ are the vertical and horizontal dimensions of the flap, $\rho$ is the density of sea water and $C_{d}=1.9$ is the drag coefficient of a plate orthogonal to the flow.

The linear frequency-domain hydrodynamic coefficients are computed using the hydrodynamic software NEMOH. $^{2}$

In the linear version of the flap WEC system, the quadratic viscous damping term is simply omitted. A linear approximation for the viscous damping term would necessitate, for each simulation, the use of an iterative linearisation procedure, referred to as spectral domain method in Folley. ${ }^{16}$

The PTO force consists of two terms. The first one is a linear damping term $-B_{P T O} \dot{x}$, and the second one is a restoring term $-K_{P T O} \dot{x}$, which, similarly to reactive control, allows the device to resonate in the incident waves. $K_{P T O}$ and $B_{P T O}$ are optimised for every $H_{m_{0}}, T_{p}$ pair in the operational space (i.e. each cell of the PM), assuming a JONSWAP spectrum with $\gamma=3.3$, resulting in a two-dimensional (2D) look-up table. The optimisation is carried out based on the linear model, by simply evaluating a range of discrete $K_{P T O}, B_{P T O}$ pairs. For each spectrum of the datasets, the $H_{m_{0}}, T_{p}$ pair of the spectrum is matched, using interpolation, with the corresponding $K_{P T O}, B_{P T O}$ pair in the look-up table.

The parameter settings which resulted in (linear) WEC angular position exceeding $45^{\circ}$ were discarded so that in some cases the 'best' parameters are sub-optimal from the point of view of power production, but enable the device to remain within meaningful physical constraints.

Finally, the linear and non-linear PMs for the flap, over its operational range $\left(H_{m_{0}} \leqslant 4 \mathrm{~m}\right)$, are shown in Figure 2. Significant differences can be observed 


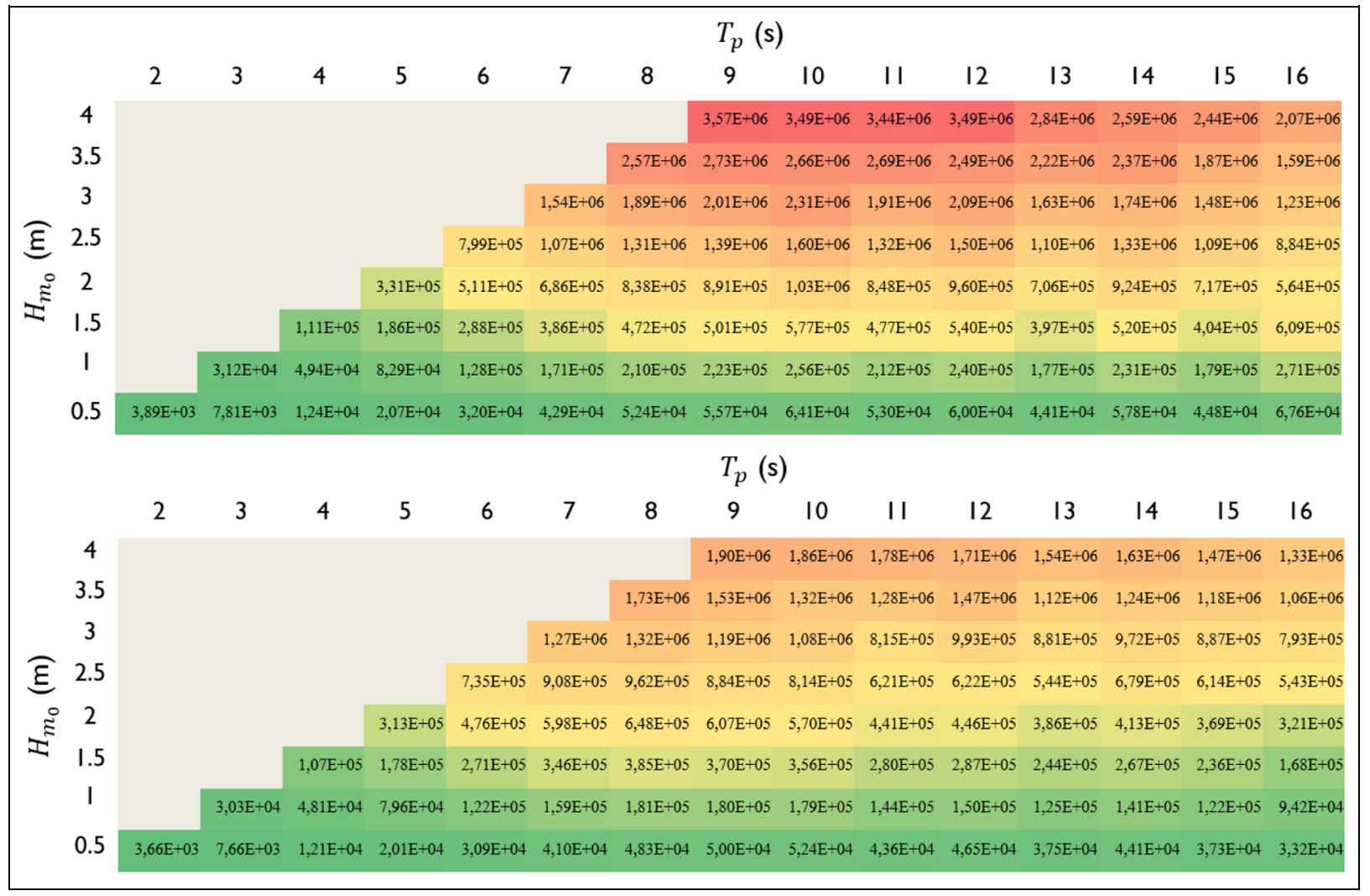

Figure 2. Linear (top) and non-linear (bottom) power matrices for the flap-type WEC (W).

between the PMs of the two models because the viscous drag term plays a significant role, especially in the most energetic sea states, where typical angular velocities are larger.

\section{Spherical heaving point absorber}

The second WEC considered is a spherical heaving point absorber, with a 5-m diameter. The motion is constrained to heave only. The buoy density is chosen to be half that of sea water so that, at rest, the position of the gravity centre is $z=0$. The radiation force $f_{r}(\dot{z}, \ddot{z})$ and diffraction forces $f_{\text {diff }}(t)$ are linearly modelled. The radiation frequency-domain coefficients $A_{r}(\omega)$ and $B_{r}(\omega)$ are computed using the hydrodynamic software NEMOH (https://heea.ec-nantes.fr/doku.php/emo/nemoh/start), as well as the diffraction transfer function.

The Froude-Krylov force, which is the sum of the static pressure force and the incident dynamic pressure force, is modelled non-linearly, taking into account the position of the device with respect to the free surface. The hydrodynamic model is then similar to the one developed in Gilloteaux, ${ }^{27}$ and used in Mérigaud et al. ${ }^{28}$ and Penalba Retes et al. ${ }^{18}$ There are, however, three significant differences with respect to the approaches used in Penalba Retes et al., ${ }^{18}$ Gilloteaux ${ }^{27}$ and Mérigaud et al.: ${ }^{28}$

- The dynamic pressure field is modified following a Wheeler et al. ${ }^{29}$ stretching approach so that the total incident pressure, measured at the free surface, is exactly zero.

- The sphere is considered to be significantly smaller than the typical wave length so that, over the sphere wetted surface, the pressure field dependence on the horizontal coordinates is omitted. Furthermore, it is considered that the intersection between the device and the free surface is always on a horizontal plane, considering that $\eta$ is everywhere equal to its value at the origin of the horizontal plane.

- The integration of the pressure over the instantaneous wetted surface is carried out analytically, as in Giorgi and Ringwood. ${ }^{30}$

The implementation, within NLFD, of the hydrodynamic model briefly described in this section, requires further steps, which are described in detail in Appendix 1.

The heaving sphere device is also subject to a quadratic viscous drag force, which depends on the relative velocity of the device with respect to the free surface, as follows

$$
f_{v}(\dot{z}-\dot{\eta})=-C_{v}(\dot{z}-\dot{\eta})|\dot{z}-\dot{\eta}|
$$

The control force, similarly to the flap-type WEC, consists of two terms, a linear damping term $-B_{P T O} \dot{z}$, and a restoring term $-K_{P T O} \dot{z}$ where $K_{P T O}$ is a 'negative stiffness' coefficient. Schematically, the role of the restoring term in the control force is to partly compensate for the natural hydrodynamic stiffness of the device so that the resonant frequency is brought closer to the peak wave period. However, since the Froude- 


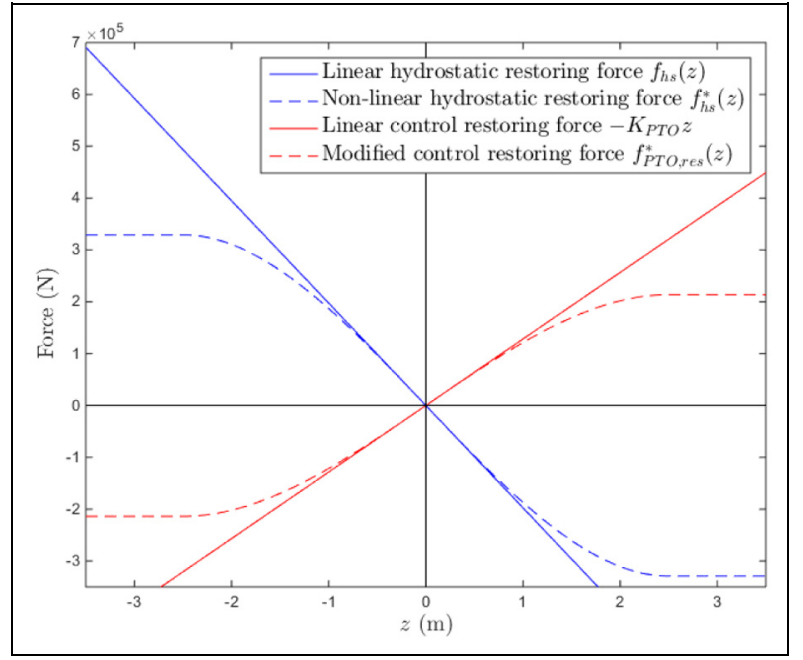

Figure 3. Linear and non-linear hydrostatic restoring force and PTO restoring force models.

Krylov forces are non-linearly modelled, the restoring term of the control is adapted, as in Mérigaud and Ringwood, ${ }^{22}$ by introducing a saturation, in a form consistent with non-linear hydrostatic restoring forces for a spherical buoy, as illustrated in Figure 3. The modified control restoring term is given as

$$
f_{\text {res }}^{*}(z)= \begin{cases}\frac{K_{\mathrm{PTO}}}{R^{2}}\left(\frac{1}{3} z^{3}-R^{2} z\right) & z \in[-R ; R] \\ -\frac{2}{3} K_{\mathrm{PTO}} R & z \geqslant R \\ \frac{2}{3} K_{\mathrm{PTO}} R & z \leqslant-R\end{cases}
$$

In this way, the desired stiffness properties around the average device position are well preserved, and the non-linear modification avoids the occurrence of unstable motions. ${ }^{22}$

In the linear version of the sphere model, no viscous drag is included, and all the hydrodynamic forces are modelled linearly, through a linear hydrostatic restoring force, a linear excitation force and linear radiation forces. Accordingly, the PTO restoring force is kept linear.

Similar to the flap case, $K_{P T O}$ and $B_{P T O}$ are optimised for each $H_{m_{0}}, T_{p}$ couple, based on the linear device model. Again, the optimisation is simply carried out through an exhaustive search approach, by assessing a whole range of parameter settings until the best combination is found.

The parameter settings which resulted in (linear) WEC motions exceeding the device dimensions were discarded so that in some cases the 'best' parameters are sub-optimal from the point of view of power production, but enable the device to remain within meaningful physical constraints.

The non-linear effects, due both to the device geometry and to the viscous drag term, are amplified under the effect of reactive control. Therefore, the non-linear device dynamics significantly affect the
WEC trajectory, resulting in the power output to be significantly smaller than what was expected from the linear model.

The adverse effect of the non-linear dynamics on power production is visible in Figure 4, by looking at the differences between the linear and non-linear PMs.

\section{Wave dataset}

\section{Wave data processing}

The WECs are studied in two locations, subject to significantly different wave conditions. It is essential to choose locations where full-wave spectra can be derived, without further assumptions about the spectral shape. The Irish Marine Institute has wave elevation measurement buoys operating at several locations off the coast of Ireland. Two of those locations were considered for this study: Belmullet, off County Mayo and Galway Bay. For each location, the Marine Institute kindly provided 1 year of wave data (http://www.marine. ie/Home/site-area/data-services/real-time-observations/ wave-buoys Belmullet buoy: Wave DataWell Wave Rider 'Berth B', Lat. 54.2339, Long. - 10.1429, year 2010; Galway Bay buoy: DataWell Wave Rider, Lat. 53.2308, Long. -9.2609, year from March 2012 to February 2013).

Although the buoys record many variables, for the purpose of this study, only free-surface elevation data were used, and processed in order to obtain 3-h spectra. Wave elevation data are organised into files corresponding to $30 \mathrm{~min}$ of wave elevation recorded at a rate of $1.28 \mathrm{~Hz}$. Unfortunately, for both locations, there is a significant proportion of missing data, roughly 30 $40 \%$, corresponding to times where the buoys were not operating properly. Therefore, not all the seasonal variability of the meteorological conditions can be reflected in the data included in this study, and thus no seasonal analysis can be carried out.

Each 30-min wave elevation data file is processed, so as to obtain a 30 -min wave spectrum estimate, in several steps:

- The $30 \mathrm{~min}$ of data are split into overlapping 3-min segments;

- In each segment, the signal is windowed through a tapered cosine function (or Tukey window) ${ }^{31}$

- The spectral density function (SDF) is estimated for each segment, by means of an FFT;

- Finally, the SDF estimates in all segments are averaged to obtain the 30-min SDF.

A few remarks are appropriate. First, the number of frequencies in the SDF is determined by the characteristics of the individual data segments. In particular, the cut-off frequency is determined as $f_{\max }=\left(1 / 2 \Delta_{t}\right)$ $=(1 / 2) f_{s}=0.64 \mathrm{~Hz}$, and the frequency step is equal to $\Delta_{f}=(1 / T)=0.0056 \mathrm{~Hz}$, where $T$ is the duration of individual data segments. Second, it was found that the 


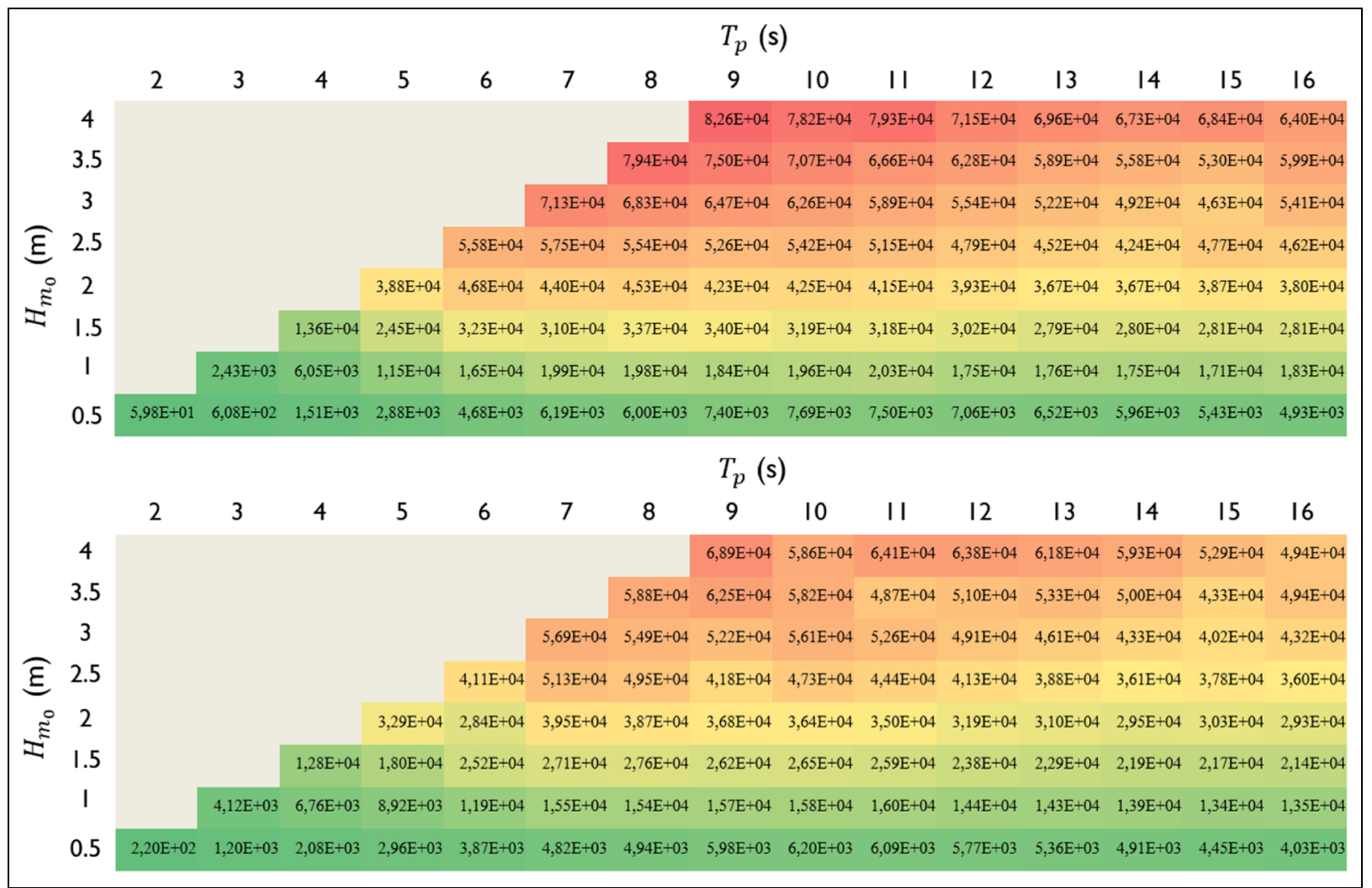

Figure 4. Linear (top) and non-linear (bottom) power matrices for the spherical WEC (W).

Table I. Number of spectra in Belmullet and Galway Bay.

\begin{tabular}{lll}
\hline Number of 3-h spectra & Belmullet & Galway Bay \\
\hline Within a year & 2920 & 2920 \\
After removing missing data and outliers & 1721 & 2046 \\
Total within operational range $\left(H_{m_{0}} \leqslant 4 \mathrm{~m}\right)$ & 1568 & 2046 \\
\hline
\end{tabular}

methodology and settings used here to produce halfhourly spectra are very similar to those used within the WaveRider measurement buoys themselves, for the same purpose (http://www.datawell.nl/Support/ Documentation/Manuals.aspx). Finally, when wave data were processed, measurement outliers can be easily detected: for example, a few consecutive measurements indicating a zero free-surface elevation clearly indicate that the buoy is not correctly operating. Similarly, a sudden 'jump' of several meters in an otherwise calm sea cannot be realistic. Such outliers always result in a significant pollution of the spectral estimate. Therefore, and since they only represented a small amount of the data, the polluted 30-min records were simply discarded from the analysis. For Belmullet, there were practically no outliers. For Galway bay, they represented less than $2 \%$ of the 30 -min spectra.

Further to the processing of the individual $30-\mathrm{min}$ record, the time resolution of the power assessment methodology was chosen as $3 \mathrm{~h}$. Indeed, too short a duration (e.g. $30 \mathrm{~min}$ ) means that individual spectra capture a lot of the inherent randomness of Gaussian seas, in addition to the meteorological variability.
Conversely, too long durations (e.g. 12h) imply that wave records, corresponding to possibly significantly different meteorological conditions, are averaged together into the same spectrum; in other words, the variability due to changes in meteorological conditions is not properly captured. For more details about the randomness of Gaussian seas, see for example. ${ }^{32}$ Even though there exists no perfect method to separate the effect of meteorological changes from the random variability of Gaussian seas (at 'constant' meteorological conditions), a 3-h duration is deemed to be an appropriate compromise, and is used in other studies, for example, De Andrés et al. ${ }^{2}$

As a result, every group of six consecutive 30-min spectra is averaged, to provide 3-h SDF estimates. Whenever one of the six spectra is missing, only the others are taken into account in the average.

Overall, although there should, in theory, be 2920 3$h$ spectra in each year, the total number of spectra in which the WECs are simulated is significantly smaller (Table 1). The main reason, by far, is missing data (the equivalent of several months in both locations). However, the number of 30-min segments discarded 


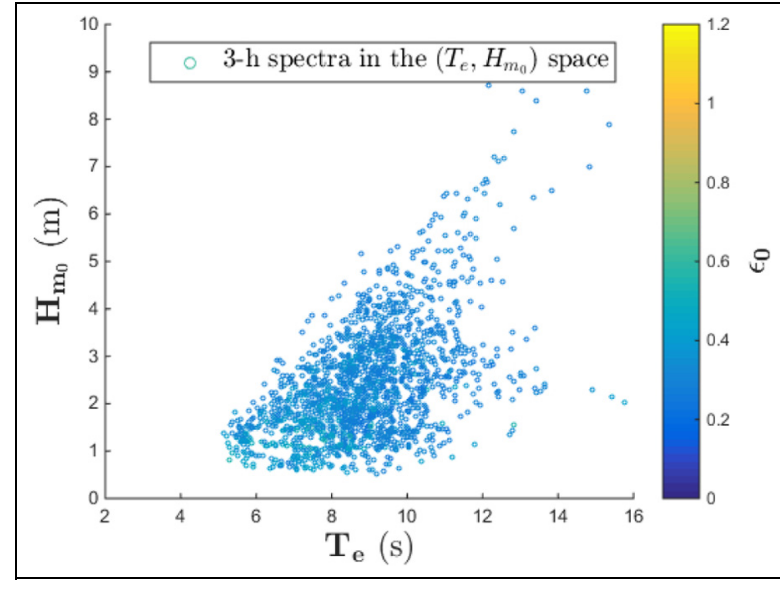

Figure 5. Scatter plot at the Belmullet location.

because of the presence of outliers in the wave timeseries had a negligible impact on the number of 3-h spectra. Finally, the operational space of the two devices is limited to $H_{m_{0}} \leqslant 4 \mathrm{~m}$, resulting in the discarding of a few additional spectra for the Belmullet location.

\section{Comparison of the wave data in the two locations}

The two chosen locations were considered for several reasons: first, measurement data are available (as stated earlier in wave data processing). Second, both locations are WEC test sites (for one-fourth scale devices in Galway bay, and for full-scale devices in Belmullet). In addition, the location in Galway Bay, sheltered by the Aran Islands, does not only result in wave spectra being less energetic than those recorded off Belmullet, but also affects typical spectral shapes. Therefore, although only considering two sites, a significant diversity of wave conditions can be observed. For a more detailed study of the wave conditions in Galway bay, see, for example, Barrett. ${ }^{33}$

The typical differences between the spectra recorded in Belmullet, and those recorded in Galway bay, are illustrated in Figures 5 and 6. Each dot on the scatter plots corresponds to a $3-\mathrm{h}$ spectrum. For each sea state, characterised by the spectrum $S(f)$, three different parameters are annotated:

- The significant wave height, here defined as $H_{m_{0}}=4 \sqrt{m_{0}}$, where $m_{0}=\in t_{0}^{\infty} S(f) d f$ (recall that, with this definition, $H_{m_{0}}$ generally differs from $H_{33 \%}$, which is the average height of the $33 \%$ highest waves. $H_{m_{0}}$ and $H_{33 \%}$ coincide only in the case of a narrowband spectrum ${ }^{23}$ );

- The mean wave energy period, defined as $T_{e}=m_{-1} / m_{0}$;

- The $\epsilon_{0}$ parameter, often referred to as broadness parameter or spectral bandwidth, ${ }^{2,19}$ computed as

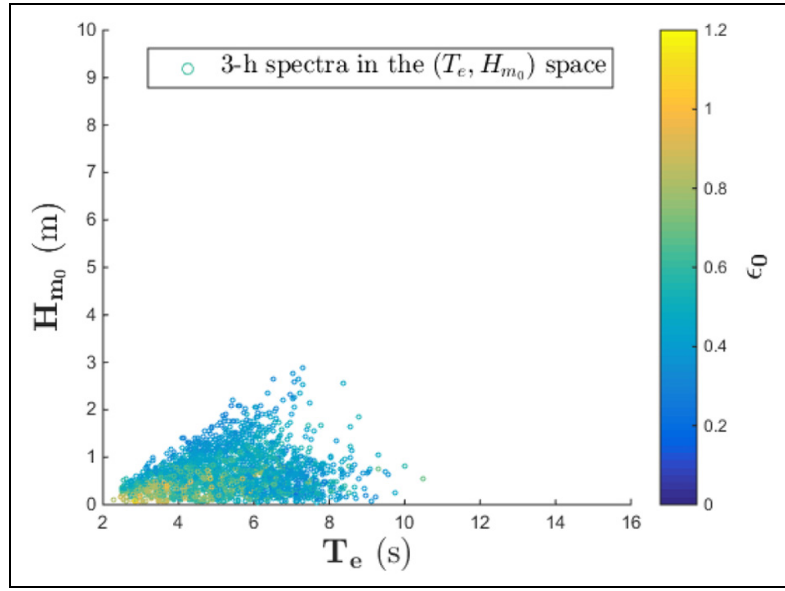

Figure 6. Scatter plot at the Galway Bay location.

$\boldsymbol{\epsilon}_{0}=\sqrt{\left(m_{0} m_{-2} / m_{-1}^{2}\right)-1}$. This latter parameter is a measure of the spectral bandwidth, which is strongly related to wave groupiness, that is, the probability to observe groups of consecutive waves bigger than a given threshold. Wave groupiness is believed to have a strong influence on WEC power production. Also note that a higher $\epsilon_{0}$ value tends to indicate a wider spreading of the spectral energy content across frequencies. Therefore $\epsilon_{0}$ has been suggested by De Andrés et al. ${ }^{2}$ and Saulnier et al. ${ }^{19}$ as an additional parameter, along with $H_{m_{0}}$ and $T_{p}$, to characterise the sea state and the WEC output more accurately.

In general, it can be seen that the sea states in Galway Bay are less energetic than the sea states in Belmullet, with typical significant wave heights being smaller by a factor of approximately 4. Furthermore, the mean energy period is also smaller in Galway compared to Belmullet, by a factor of approximately 2 .

The $\epsilon_{0}$ parameter, however, is in general significantly bigger in Galway Bay than in Belmullet, which can be explained by the spectra in Galway Bay having more various shapes than the ones in Belmullet, due in particular to the presence of the Aran Islands. Therefore, the spectra observed in Galway Bay are not simply a downscaled version of the Belmullet ones; they are also significantly distorted with respect to standard, uni-modal spectral shapes - which generally have a small $\epsilon_{0}$ value.

It is also interesting to observe that there is generally an inverse correlation between the level of energy in the sea states (here characterised by the significant wave height) and $\epsilon_{0}$. This can be explained by the fact that fully developed sea states, which are the most energetic and are better described by means of parametric spectral shapes, have a small $\epsilon_{0}$ value, most of the spectral energy being strongly concentrated around the spectrum peak frequency. 


\section{Number and length of simulations per sea state}

\section{Number of simulations and computational requirements within the NLFD framework}

An important aspect of WEC power production assessment, neglected in most studies (with the notable exception of Saulnier et al. ${ }^{34}$ and other works by the same author), is the number and length of the simulations, necessary to obtain power output estimates, with a desired confidence interval.

Indeed, a finite-length realisation of a given sea spectrum can result in power estimates which significantly depart from the long-term average power output. Longer simulations, or equivalently a larger number of them, provide a more accurate power estimate, at the expense of more computational effort. It is therefore necessary to find the proper trade-off between the estimate accuracy and the corresponding computational effort, taking into account the specific requirements of each study.

In this study, the simulation method used, NLFD, has very specific implications. Due to the solution method, two main parameters affect the computational time of one given simulation: the number of iterations before convergence is achieved, and the central processing unit (CPU) time required by each iteration. The latter, due to the computation of the gradient of the nonlinear forces, depends on the size of the problem in $N^{2} \ln (N)$, where $2 N \Delta_{t}=T$ is the duration of the simulated, periodic signal. Therefore, while it is statistically equivalent to run one simulation of length $T=1000 \mathrm{~s}$, or to run 10 simulations of length $T=100 \mathrm{~s}$, the two situations differ with respect to the computational time: if the sampling time is $\Delta_{t}=1 \mathrm{~s}$, the computational time in the first situation is proportional to $1000^{2}$ $\ln (1000)=3 \ln (10) \cdot 10^{6}$ while, in the second situation, it is only proportional to $10 \times 100^{2} \ln (100)=$ $2 \ln (10) \cdot 10^{5}$.

As a consequence, using NLFD makes it preferable to run many, relatively short, rather than fewer, large simulations. However, excessively short simulations imply that $\Delta_{f}=1 / T$ is not refined enough so that the frequency content of the spectrum may be poorly resolved.

Finally, two parameters must be set in a sensible way: the length of each simulation, which guarantees an accurate frequency discretisation, and the number of simulations, which reduces the uncertainty on power estimates.

\section{Length of the simulations}

The issue of the simulation length is investigated in detail, considering a variety of JONSWAP spectra with a range of $\left(H_{m_{0}}, T_{p}\right)$ pairs spanning the operational range of the WECs considered. JONSWAP spectra are a conservative choice, since their energy is sharply concentrated around the peak frequency, and therefore the sensitivity of the power estimates to the frequency discretisation can be expected to be stronger than in spectra with a larger bandwidth.

For each spectrum, various signal durations $T$ are considered. For every $T$, the average power is estimated for both linear and non-linear WEC models (running a large number of simulations in the latter case). The results of this convergence study are shown in Figure 7.

For the linear models, the sensitivity of the power estimate to the simulation length (i.e. to the refinement of the frequency discretisation) is more pronounced than for the non-linear models. However, linear computations being computationally cheap, a relatively long simulation time $(600 \mathrm{~s})$ can be chosen. The results of the non-linear models are less sensitive to the frequency discretisation. A period of $200 \mathrm{~s}$ is chosen, allowing for accurate power estimates while preserving the computational benefits of the NLFD simulation method.

\section{Methodology to determine an appropriate number of simulations}

After choosing a given simulation length, an adequate number of simulations per sea state must be found. The appropriate answer depends on the method through which the wave elevation time-series are randomly generated. As highlighted in Saulnier et al. ${ }^{34}$ and further studied in Mérigaud and Ringwood, ${ }^{32}$ there are two harmonic superposition methods to generate random, finite-length samples from a target sea spectrum:

- In the 'deterministic-amplitude scheme' (DAS), a set of random phases $\phi_{k}$ is drawn, and the timeseries is generated as

$\eta(t)=\sum_{k=1}^{N} A_{k} \cos \left(\omega_{k} t+\phi_{k}\right)$

- With DAS, each generated signal contains the exact same amount of energy, equal to $m_{0} T$. This method is not theoretically correct since it does not reproduce the actual variability of a Gaussian process sampled over a finite duration. Nevertheless, in the context of WEC power calculations, the small variance of the average power, from one simulation to the next, allows for using relatively few simulations to obtain an accurate estimate for the long-term average power. However, the fact that DAS departs from the theoretical statistical properties of actual Gaussian seas may introduce a bias in non-linear power computation results ${ }^{32,34}$ although it does not seem to be the case for the non-linear WEC model studied in Saulnier et al. ${ }^{34}$ Therefore, preliminary tests should be carried out, prior to choosing DAS, to ensure that the resulting power estimates are not significantly biased. 


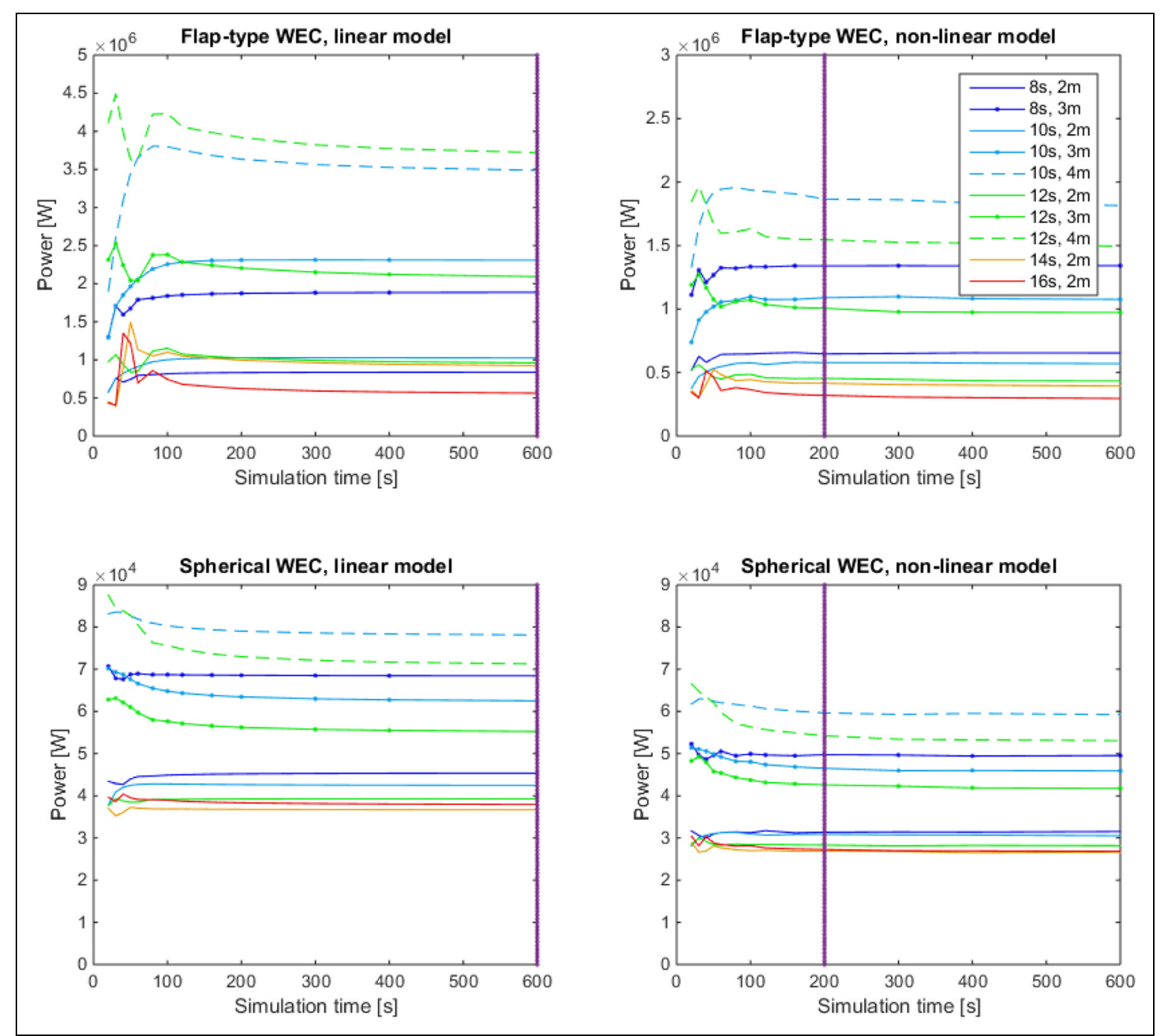

Figure 7. Convergence studies to determine appropriate simulation lengths, for JONSWAP spectra with various $\left(H_{m_{0}}, T_{p}\right)$ values.

- The 'random-amplitude scheme' (RAS) is the theoretically consistent simulation scheme. ${ }^{35}$ The formulation is similar to equation (13), except that the $A_{k}$ coefficients, instead of being deterministic, are randomly generated following a Rayleigh distribution with variance $2 S\left(\omega_{k}\right) \Delta_{f}$. With RAS, the generated signals do not all contain the same amount of energy, reproducing accurately the statistical properties of a Gaussian sea surface elevation process recorded over a finite duration. Although RAS is perfectly realistic, the results of individual simulations are significantly more variable than with DAS, thus requiring a larger number of simulations to achieve accurate power estimates. Hence, DAS is preferable in most cases, provided that DAS results are not biased. Nevertheless, in other applications such as extreme design loads, a proper stochastic representation (RAS) is essential.

A preliminary study was carried out in order to determine what time-series generation method was preferable, and how many simulations were necessary to achieve accurate estimates of the average power from each spectrum. The methodology retained is to make decisions in a conservative manner, based on an 'unfavourable' spectrum with respect to the accuracy of power estimates, that is, a spectrum for which the signal energy varies a lot from one simulation to another.

For a wave spectrum $S(f)$, and for signals with duration $T$ longer than a few tens of seconds, the average power of the wave signal, estimated over $T$, has a variance approximately equal ${ }^{34}$ to

$$
\sigma^{2}\left[\hat{m}_{0}\right]=\frac{1}{T} \int_{0}^{\infty} S(f)^{2} d f
$$

In addition, it can be assumed that more variance in the energy content of the generated wave signals (large $\left.\sigma^{2}\left[\hat{m}_{0}\right]\right)$ implies more variance in the WEC power output estimate (large $\sigma^{2}[\hat{P}]$ ), which can be justified in a simple manner: indeed, a more or less energetic wave signal is likely to result in a similarly higher or lower WEC power output. Therefore, for a given spectrum 
Table 2. Mean and variance of power estimates, and $95 \%$ confidence interval (in $\%$ of the mean) for $N=10$ and $N=50$ simulations of $200 \mathrm{~s}$.

\begin{tabular}{lllll}
\hline & Flap & & \multicolumn{2}{l}{ Sphere } \\
\cline { 2 - 3 } & DAS & RAS & DAS & RAS \\
\hline$\mu[\hat{P}]$ & $1.73 \times 10^{6}$ & $1.71 \times 10^{6}$ & $5.43 \times 10^{4}$ & $5.40 \times 10^{4}$ \\
$\sigma^{2}[\hat{P}]$ & $2.82 \times 10^{9}$ & $2.19 \times 10^{11}$ & $9.7 \times 10^{5}$ & $1.45 \times 10^{8}$ \\
$95 \% \mathrm{Cl}(N=10)$ & $\mu \pm 2.2 \%$ & $\mu \pm 19 \%$ & $\mu \pm 1.3 \%$ & $\mu \pm 15 \%$ \\
$95 \% \mathrm{Cl}(N=50)$ & $\mu \pm 0.9 \%$ & $\mu \pm 7.8 \%$ & $\mu \pm 0.5 \%$ & $\mu \pm 6.3 \%$ \\
\hline
\end{tabular}

DAS: deterministic-amplitude scheme; RAS: random-amplitude scheme.

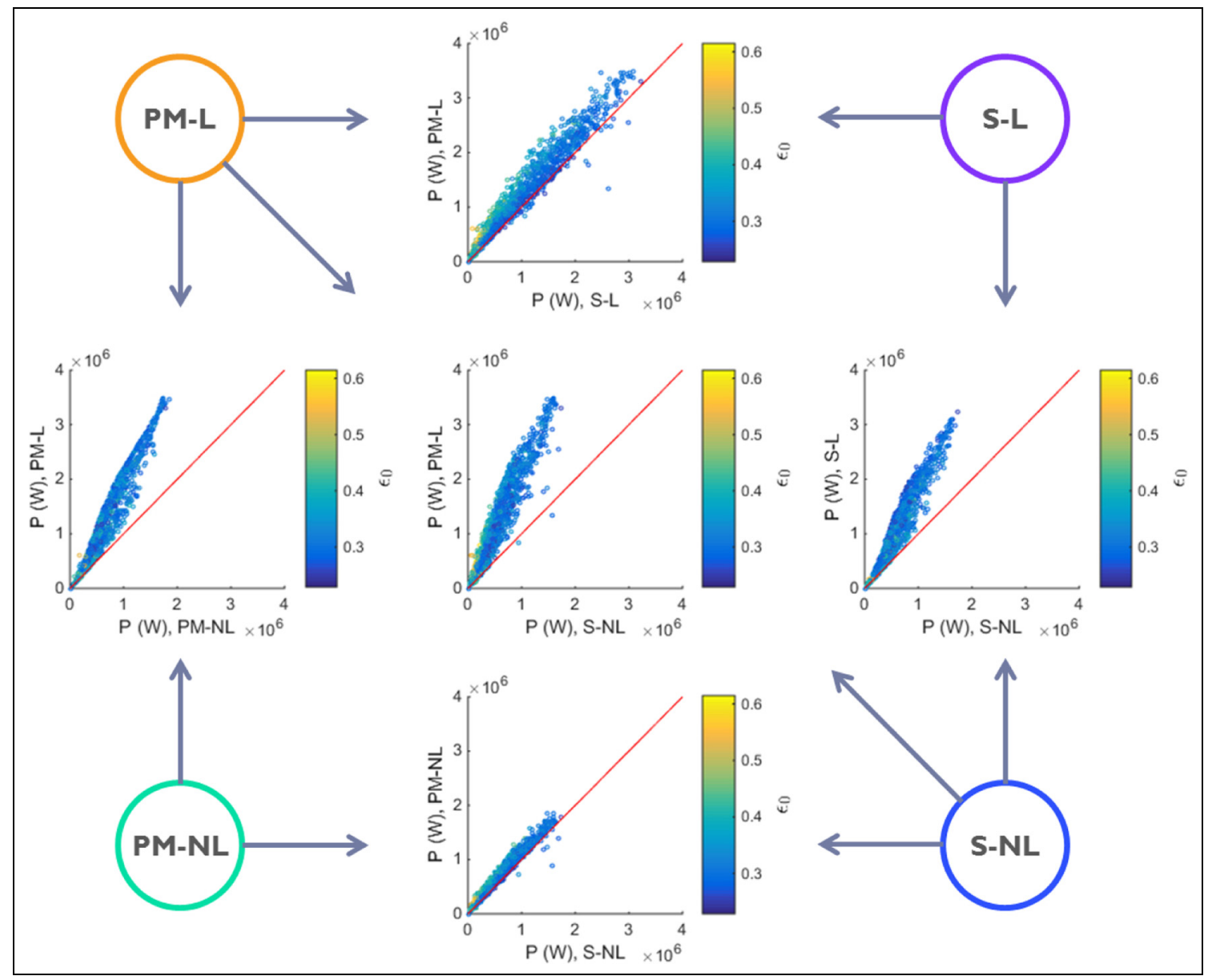

Figure 8. Flap, Belmullet.

$S(f)$, if the energy content of generated free-surface signals presents a large variability from one simulation to another, so will the WEC power output.

Given equation (14), the spectra which present the highest $\sigma^{2}\left[\hat{m}_{0}\right]$ are both the most energetic, and amongst them, the ones whose energy content is sharply concentrated around a peak frequency. Energetic sea states with a sharply concentrated energy content are fully developed ones, and are well described by means of standard parametric spectral shapes. Therefore, choosing a JONSWAP spectrum with a high $H_{m_{0}}$ value guarantees that $\sigma^{2}\left[\hat{m}_{0}\right]$ is high. Finally, it is found that, for the same $H_{m_{0}}$, JONSWAP spectra with higher $T_{e}$ values have a larger $\sigma^{2}\left[\hat{m}_{0}\right]$. Based on those simple qualitative arguments, and given the operational space considered in this study (Figures 5 and 6), a JONSWAP spectrum with $T_{p}=12 \mathrm{~s}$ and $H_{m_{0}}=4 \mathrm{~m}$ is believed to represent an appropriate spectrum for the preliminary study.

For the chosen JONSWAP spectrum, 1000 simulations of duration $T=200 \mathrm{~s}$ were run for the non-linear flap-type WEC and for the non-linear spherical heaving point absorber, both with RAS and DAS. In each case, the average power value and the variance of the power 
Table 3. Numerical performance of non-linear power assessment (S-NL).

\begin{tabular}{lll}
\hline & Flap & Sphere \\
\hline Nb of spectrum per loc. & $\sim 2000$ & $\sim 2000$ \\
Nb of sim. per loc. & $\sim 20,000$ & $\sim 20,000$ \\
$t_{C P U} /$ sim. (NLFD) & $\sim 0.006 \mathrm{~s}$ & $\sim 0.09 \mathrm{~s}$ \\
$t_{C P U} /$ loc. (NLFD) & $\sim 2 \mathrm{~min}$ & $\sim 3 \mathrm{~min}$ \\
$t_{C P U} /$ sim. (RK2) & $\sim 2.4 \mathrm{~s}$ & $\sim 2.4 \mathrm{~s}$ \\
Estimated $t_{\text {CPU }} /$ loc. (RK2) & $\sim 13 \mathrm{~h} 00$ & $\sim 13 \mathrm{~h} 00$ \\
\hline
\end{tabular}

CPU: central processing unit; NLFD: non-linear frequency-domain; RK2: second-order Runge-Kutta.

estimate were evaluated, as shown in Table 2. Using basic statistical results, one can infer the half-width of the $95 \%$ confidence interval for various numbers of simulation runs. In Table 2 , the $95 \%$ confidence interval is shown as a percentage of the average, for $N=10$ and $N=50$. Since DAS does not seem to introduce any significant bias, it is found preferable to generate the time-series using DAS rather than RAS. With DAS, choosing $N=10$ provides estimates with sufficient accuracy (for both devices, the half-width of the $95 \%$ confidence interval is smaller than $2.5 \%$ ). In contrast, although using RAS is theoretically more realistic, it would require a large number of simulations to obtain accurate power estimates.

Finally, note that the above considerations, concerning the ideal number of simulations, are only relevant to the simulation of non-linear WEC models. Indeed, as shown in Mérigaud and Ringwood, ${ }^{32}$ DAS, applied for the simulation of a linear WEC model, always results in the same power value, which is the long-term average. Therefore, just one single DAS simulation is necessary to estimate the long-term average power for a linear WEC model.

\section{Numerical results}

\section{Numerical performance}

For each of the two sites and two WEC systems considered, power production assessment is carried out in four different ways: PM-L, PM-NL, S-L and S-NL. All the non-linear computations, that is, to fill-in the non-linear PMs, and to compute the power output in every individual sea state with the non-linear models, are carried out using the NLFD method.

For each location, the number of 3-h spectra is of the order of 2000. The PM-L and PM-NL approaches only necessitate interpolation of each $H_{m_{0}}, T_{p}$ couple

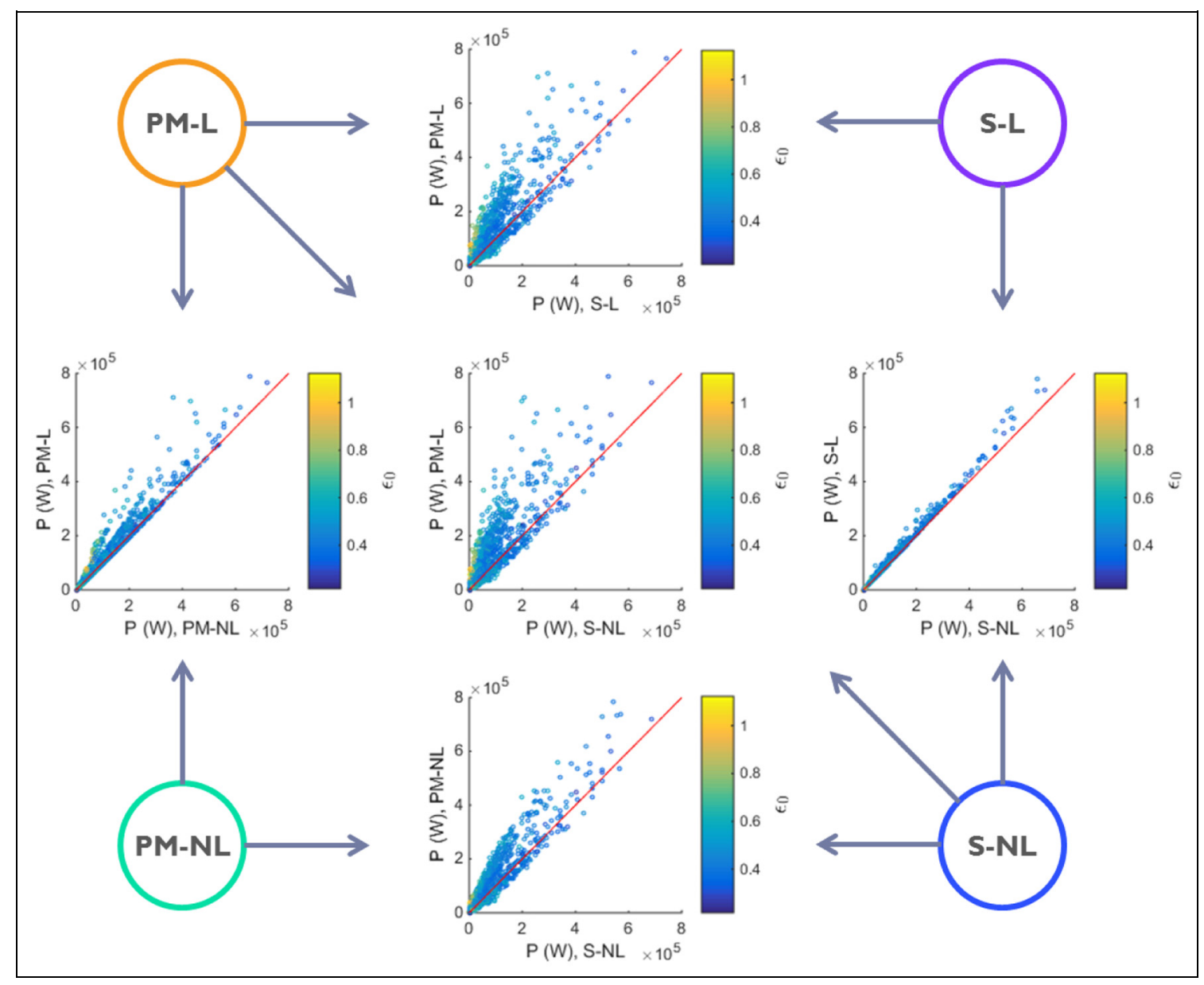

Figure 9. Flap, Galway Bay. 


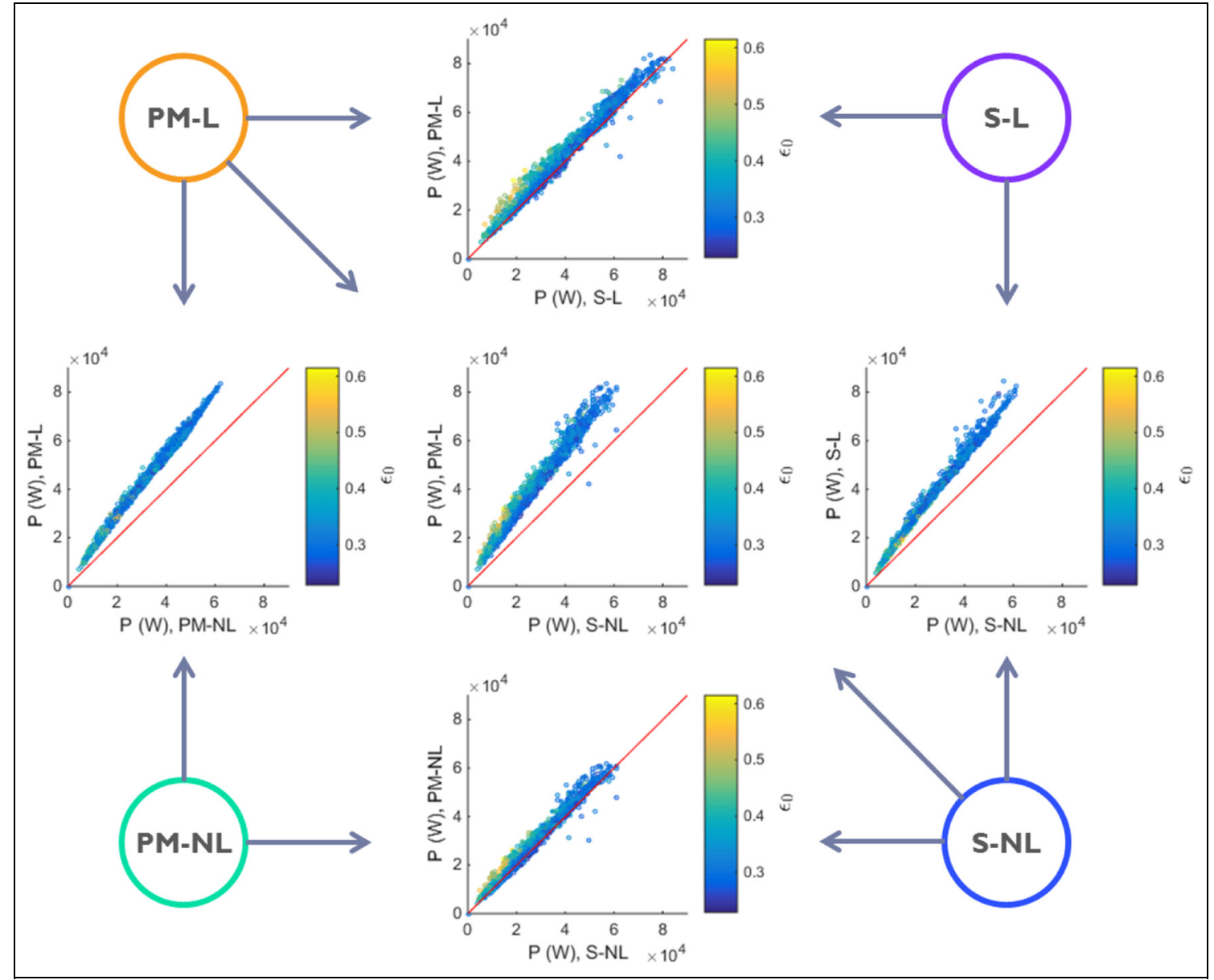

Figure I0. Sphere, Belmullet.

in the linear and non-linear PMs, respectively, while for the S-L approach, the average power output is determined using only one single realisation of the sea state. Therefore, none of the three methods necessitates more than a couple of seconds to cover the whole dataset at each location (All numbers concerning the computational performance are given for a $3.5 \mathrm{GHz}, 8$-core Intel ${ }^{\circledR}$ process).

For the S-NL case (Table 3), the number of 200-s simulations run for each spectrum is 10 , as determined in the preliminary study detailed in section 'Number and length of simulations per sea state'. The total number of NLFD simulations for each location and WEC system is then of the order of 20,000. For the flap, the NLFD method takes only approximately 2 min to cover the whole range of simulations for each location. The time necessary to carry out the 20,000 simulations for the spherical point absorber is slightly higher, of the order of $3 \mathrm{~min}$, which remains relatively modest.

The reason why the NLFD simulations are slower for the sphere than for the flap is that, in the sphere case, more iterations are needed for the Newton method to converge, due to more significant non-linearities.
Overall, using NLFD computations, S-NL appears to be within easy computational reach, while overcoming the limitations of PM-L, PM-NL, and S-L. A few time-domain simulations were run for the same WEC models, using a simple RK2 scheme with direct integration of the radiation force convolution, and the time per simulation was estimated to be higher than $2 \mathrm{~s}$ (for an integration time step of $0.01 \mathrm{~s})$. The total time necessary to perform the 20,000 simulations in each sea state would then have been at least $13 \mathrm{~h} 00$. More efficient time-domain simulation methods could be chosen, by resorting to a state-space approximation of the radiation terms. ${ }^{36}$ However, unlike NLFD, the resulting computational gains would be at the expense of the solution accuracy.

\section{Detailed results per location and WEC system}

Power assessment results are detailed for each site and WEC system, in Figures 8-13. Recall that the reference point of comparison, to assess the accuracy of all other methods, is the S-NL configuration, where the device is modelled non-linearly and simulated in every individual sea state, using NLFD. 


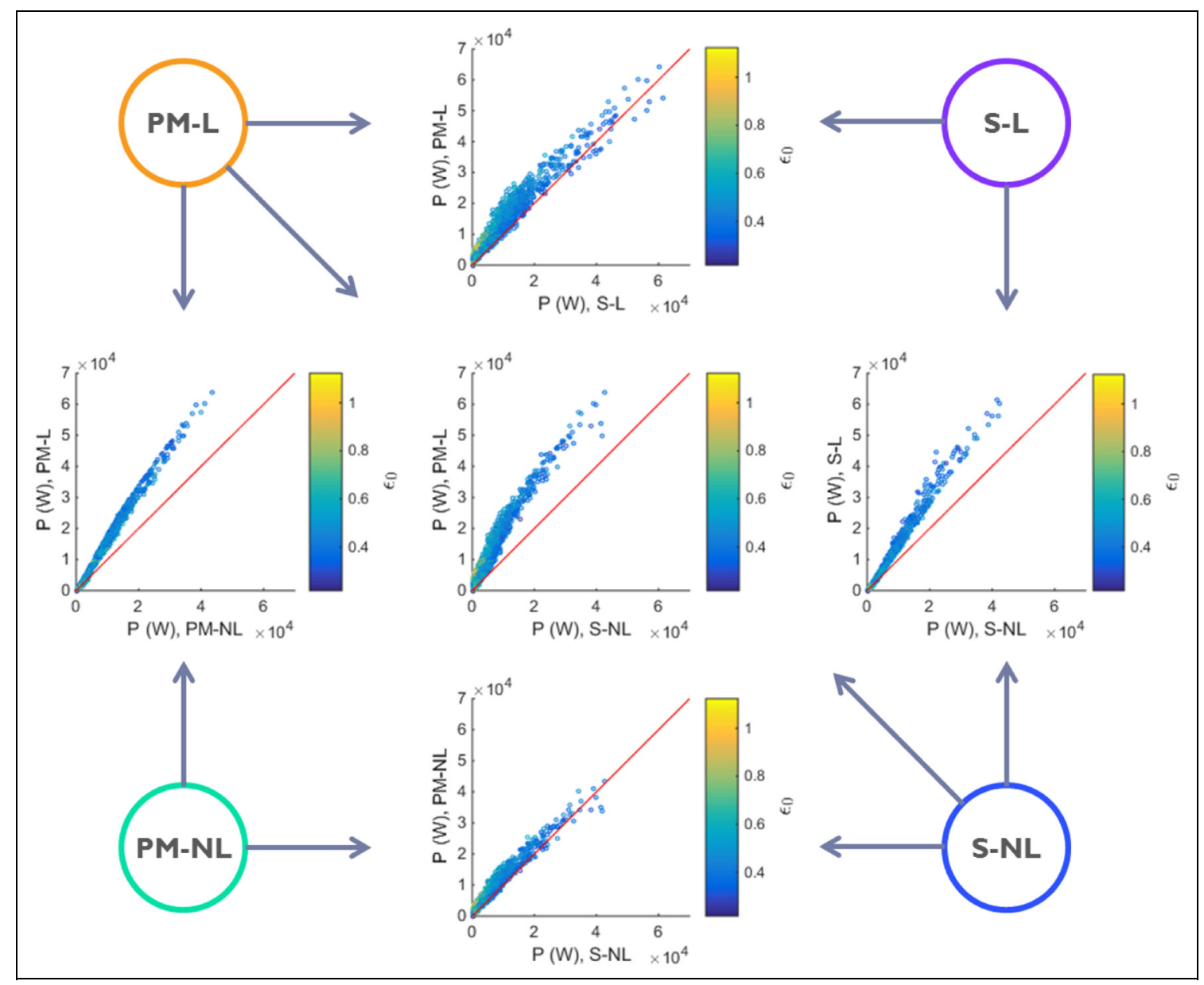

Figure I I. Sphere, Galway Bay.

In Figures 8-11, relevant comparisons between the four power assessment methods (PM-L, PM-NL, S-L and S-NL) are visualised by means of scatter plots, in a way identical to the results presented in De Andrés et al. ${ }^{2}$ Each scattered point represents one 3-h spectrum, and has horizontal and vertical coordinates representing the power output computed through the two methods being compared. Therefore, the distance between each point and the diagonal axis represents the discrepancy between the power output computed using the two different methods. Furthermore, the colour indicates the value of the $\epsilon_{0}$ parameter of the spectrum considered.

By looking at the comparison between the figures obtained through a PM, and those resulting from computations in actual wave spectra (scatter plots of PM-L against S-L, and PM-NL against S-NL), for all devices and locations, it can be seen that the error due to the PM representation is significant for both devices and locations, and tends to be larger for wave spectra having a larger $\epsilon_{0}$ parameter. Such a correlation between the $\epsilon_{0}$ parameter and the error resulting from the PM approximation is consistent with the results shown in De Andrés et al. ${ }^{2}$ Since the range of $\epsilon_{0}$ values is much more restricted in Belmullet, the effect of the spectral shape is proportionally more visible in Galway Bay, as can be seen by considering the 'PM-NL' error bars in Figure 13.

Interestingly, in Figures $8-11$, the top and bottom plots look relatively similar to each other, which implies that the errors due to the use of a PM are similar, whether the device is linearly or non-linearly modelled. The same goes for the left- and right-hand side plots, suggesting that the errors due to linear WEC modelling remain similar, whether the WEC power output is computed through a PM or using actual spectra. In each of the four figures, the central graph illustrates the overall errors, encountered using a linear approximation of the WEC dynamics, and an excessively simplified sea-state representation through a PM.

For the flap, in Belmullet (Figure 8), the errors due to the use of a PM are significant, resulting in overestimating the average annual power by more than $20 \%$ (Figures 12 and 13). The errors due to the linear device modelling are, however, of an even larger order of magnitude, and are increasingly significant in more energetic sea states (PM-L/PM-NL and S-L/S-NL comparisons on Figure 8) where viscous effects are 


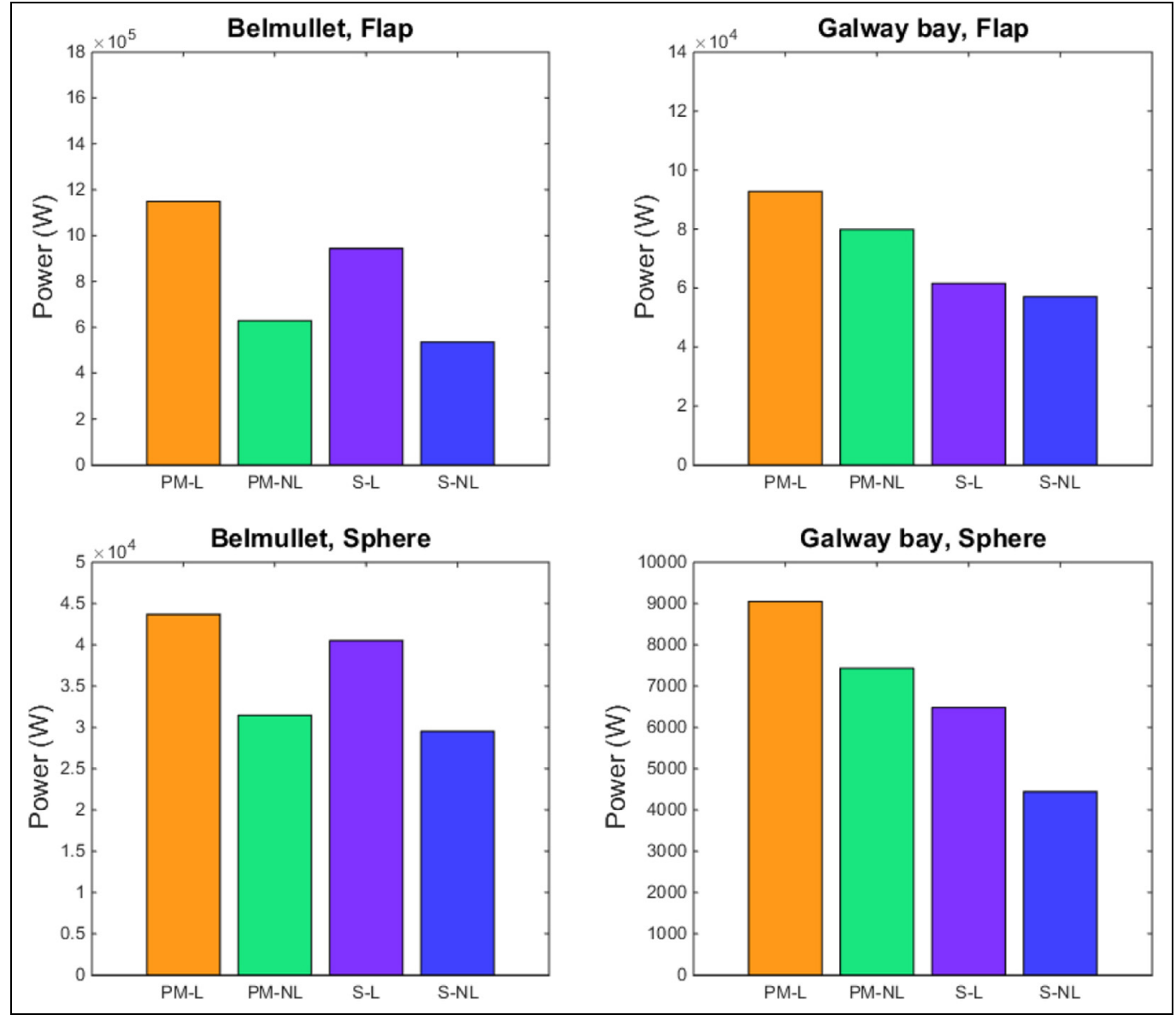

Figure 12. Annual power production for the different WECs, locations, and WEC representations.

amplified. The resulting average power overestimation is of approximately $60 \%$.

In Galway Bay, where the sea states are less energetic, the effect of viscous drag is less significant, resulting in linear results matching the non-linear with reasonable accuracy (Figures 9, 12 and 13). In contrast, the effect of describing the flap device by means of a PM, whether it be linearly or non-linearly evaluated, has a clearly visible impact on power assessment, both on a sea state by sea-state basis (Figure 9) and on an annual basis (approximately 35\% overestimation of the average annual power, as shown in Figures 12 and 13).

For the spherical point absorber, in Belmullet, it can be seen in Figures 10, 12 and 13 that the errors, due to a linear approximation of the device dynamics, are approximately five times larger $(\approx 35 \%)$ than those committed using a PM instead of computing the WEC output in actual sea states $(\approx 7 \%)$.

In Galway Bay, the two error sources are of a similar order of magnitude: PM-NL results in overestimating the annual power output by $25 \%-30 \%$, while S-L yields an overestimation in the order of $45 \%$. The error encountered by accumulating the two sources of errors, that is, with PM-L, is more than the sum of the errors resulting from the two approximations considered separately.
The reason why the discrepancies, between the linear and non-linear model results, are so significant for both devices is that the reactive control strategy enables the WECs to resonate in all sea states, including those that are less energetic, thus magnifying the non-linearities due to the device geometry or to quadratic viscous drag. The latter is particularly significant for the flap in the Belmullet location.

The control strategy may also heighten the errors due to the use of a PM, especially in Galway Bay. Indeed, with the PTO stiffness term, the devices both become of a resonant type, particularly sensitive to excitation forces around a design frequency. The PTO parameters are optimised, assuming a JONSWAP spectral shape - for which the energy is sharply concentrated around the peak frequency - while actual spectra in Galway Bay present a much larger spread of wave energy across frequencies. Therefore, the power output is significantly smaller in actual wave spectra than assuming a standard JONSWAP spectrum.

Finally, in order to ensure that the conclusions of this study are not specific to the chosen control method, power production assessment was also carried out for the same two devices and locations, this time using a simple passive linear damper, optimised for every $H_{m_{0}}, T_{p}$ condition. Only the error in terms of annual 


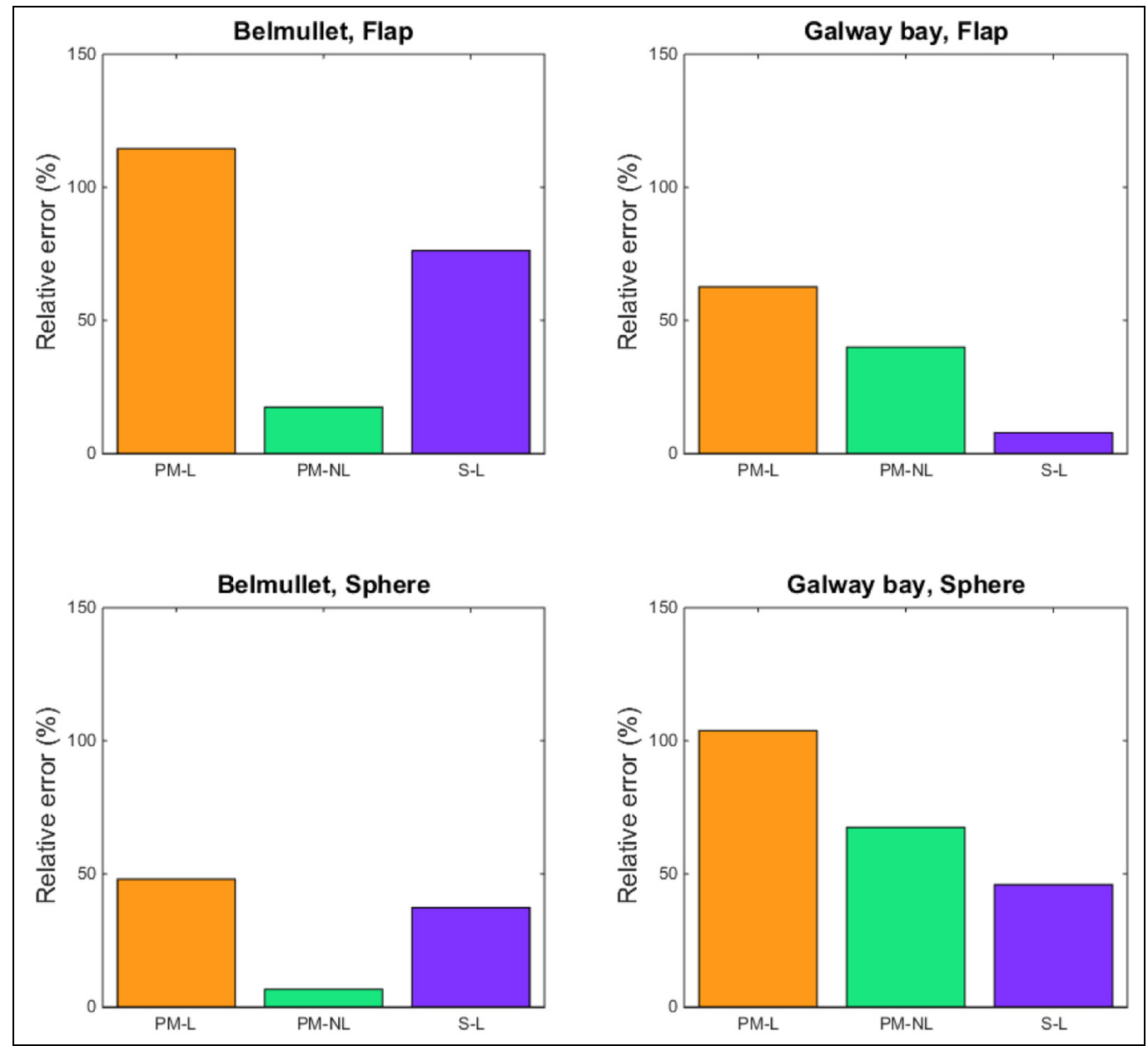

Figure 13. Relative error in annual power production (with respect to S-NL results).

average power is shown in Figure 14. It can be observed that although the two types of errors are significantly smaller than in the case with reactive control, both can be significant. The errors due to the PM representation are larger in Galway Bay (approximately 20\%) than in Belmullet (approximately 7\%). In contrast, the errors due to the inaccurate description of the WEC dynamics are more important in Belmullet than in Galway Bay, due to the occurrence of more energetic sea states.

\section{Discussion and conclusion}

\section{Number and length of simulations}

The issue of the number and length of simulations per sea state deserves to be seriously addressed in every power production assessment study. RAS simulations are theoretically correct, since they exhibit the actual properties of a finite-length realisation of a Gaussian process. However, using RAS simulations can result in a large variance of the power estimates, thus requiring an accordingly large number of simulations to obtain an estimate with acceptable accuracy. DAS, in contrast, results in a significantly smaller variance of the power estimates. Therefore, although DAS is not theoretically consistent with Gaussian process theory, the method can be useful to obtain power estimates within a reasonable number of simulations. Nevertheless, it has to be ensured that DAS does not produce a biased power estimate.

As a consequence, it is recommended that a preliminary study is systematically carried out based, for example, on a JONSWAP spectrum $S^{*}(f)$ with high $H_{m_{0}}$ and $T_{p}$ values, which constitutes an unfavourable case from the point of view of the power estimate variance. The output of such a preliminary study could consist of:

- A choice of method (RAS or DAS), DAS being justified only if it does not seem to introduce any significant bias;

- A number of simulations - for a fixed simulation length;

- For the chosen number of simulations, a conservative estimate of the uncertainty on power calculations, for example the half-width of the $95 \%$ confidence interval - obtained with the spectrum $S^{*}(f)$. 


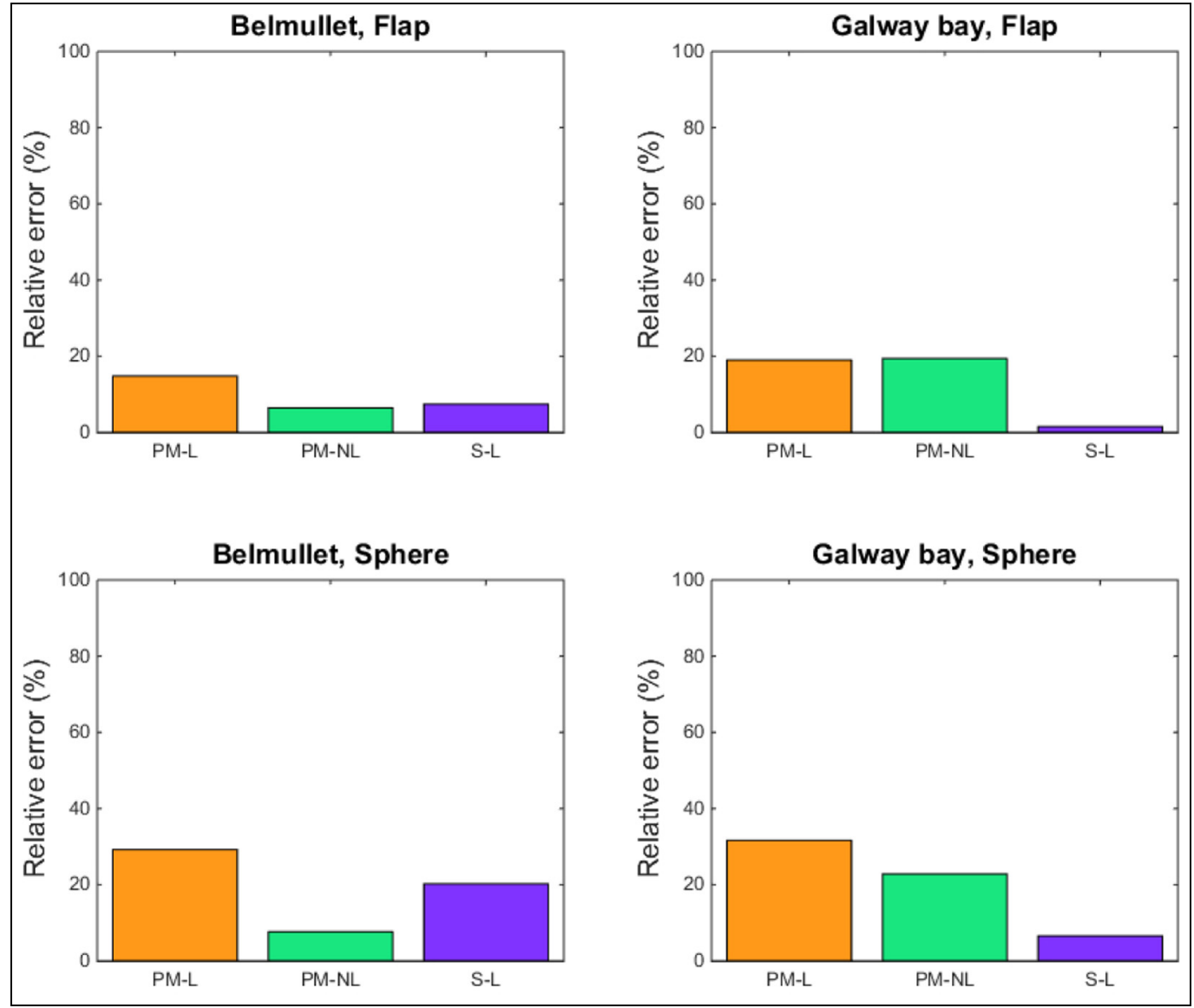

Figure 14. Relative error in annual power production (with respect to S-NL results), with passive linear damping.

\section{The PM and power assessment errors}

Even with only two WECs and two locations, a wide range of scenarios in terms of power production assessment can be considered.

For some locations and WEC types, PM may result in a relatively good approximation of power production, for example in the Belmullet location for the controlled sphere. However, that is not true anymore for locations where spectra depart significantly from standard spectral shapes, in which case a more refined approach, such as simulation in every individual spectrum, is necessary.

Consistent with the results of De Andrés et al., ${ }^{2}$ a larger spectral spreading often implies a larger error due to the PM approach. Nevertheless, even in locations where spectra have a limited spreading, the errors can be significant.

For resonant-type devices, which deliver better output when wave energy is sharply concentrated around a specific frequency, the errors related to the PM can be particularly large, resulting in a significantly overestimated power assessment, both in individual sea states and on an annual basis. As a consequence, the presence of reactive control, which tends to make the device resonant, heightens the errors induced by the PM representation.

However, even for devices which are not controlled, in locations with relatively low energy and large $\epsilon_{0}$ values, the approximation due to the PM can become the most significant source of error (such as the two uncontrolled device in Galway Bay).

Regardless of the wave spectral shape, describing non-linear system dynamics accurately is essential for power assessment. Non-linear effects become particularly significant in energetic sea states, or when the device undergoes large amplitude motion under the effect of power-maximising control.

In the cases presented in this study, both the linear WEC dynamical representation and the sea-state description through a PM tend to yield an overestimation of the WEC output, compared to more realistic calculations. Therefore, the errors due to the two approximations sum, and result in even larger errors, visible in the comparisons between PM-L and S-NL. However, situations could be imagined, where the two sources of approximation offset each other, thus minimising the overall error.

In summary, the errors encountered, using a PM representation on one hand, and describing the system dynamics linearly on the other hand, can both be significant, and of a similar order of magnitude. The presence of control heightens all the differences observed; however, both types of error remain significant in uncontrolled conditions.

Therefore, it is essential to improve upon the PM approach, while preserving the accuracy of the system 
dynamical description, which is made possible by the existence of appropriate simulation tools.

\section{NLFD as a suitable tool for fast and accurate power assessment}

As stated in sections 'Introduction' and 'NLFD simulation', NLFD is significantly faster than RK2 timedomain integration, without resulting in any important approximation of the WEC dynamics.

Although other computation methods can be considered, NLFD simulations, based on a well-calibrated number of relatively short simulations for each individual sea state of the dataset, can make the S-NL approach computationally feasible, which overcomes the drawbacks of both PM-NL and S-L in terms of accuracy. For velocity-dependant non-linearities, such as quadratic viscous drag, iterative linearisation procedures are also interesting candidates. ${ }^{16,22}$

Alternatively to simulations in every sea state, other approaches, such as the MaxDiss selection technique, ${ }^{2}$ can also help refining power estimates, with the limitation that the resulting device representation is site-specific. Combining the MaxDiss selection technique with NLFD simulations could interestingly cumulate the computational advantages of both methods.

Finally, let us stress that the length of the dataset (1 year) considered here, and the fact that a significant fraction of the data are missing, makes the dataset insufficient for accurate WEC power assessment, which would require significantly longer periods, covering the wave resource variability on both seasonal and annual scales. However, the relative benefits of the proposed approach, in terms of accuracy and computation time, generalise to longer datasets.

\section{Declaration of Conflicting Interests}

The author(s) declared no potential conflicts of interest with respect to the research, authorship and/or publication of this article.

\section{Funding}

The author(s) disclosed receipt of the following financial support for the research, authorship, and/or publication of this article: This article is based upon work supported by Science Foundation Ireland under Grant No. $12 / \mathrm{RC} / 2302$ for the Marine Renewable Ireland (MaREI) centre.

\section{References}

1. Babarit A, Hals J, Muliawan M, et al. Numerical benchmarking study of a selection of wave energy converters. Renew Energ 2012; 41: 44-63.
2. De Andrés A, Guanche R, Weber J, et al. Finding gaps on power production assessment on WECs: wave definition analysis. Renew Energ 2015; 83: 171-187.

3. Rusu L and Onea F. Assessment of the performances of various wave energy converters along the European continental coasts. Energy 2015; 82: 889-904.

4. Rusu L and Onea F. The performance of some state-ofthe-art wave energy converters in locations with the worldwide highest wave power. Renew Sust Energ Rev 2017; 75: 1348-1362.

5. Rusu E and Onea F. Estimation of the wave energy conversion efficiency in the Atlantic Ocean close to the European islands. Renew Energ 2016; 85: 687-703.

6. Silva D, Rusu E and Soares CG. Evaluation of various technologies for wave energy conversion in the Portuguese nearshore. Energies 2013; 6(3): 1344-1364.

7. Carballo R and Iglesias G. A methodology to determine the power performance of wave energy converters at a particular coastal location. Energ Convers Manage 2012; 61: 8-18.

8. Robertson B, Hiles C, Luczko E, et al. Quantifying wave power and wave energy converter array production potential. Int J Mar Energ 2016; 14: 143-160.

9. Carballo R, Sánchez M, Ramos V, et al. The intraannual variability in the performance of wave energy converters: a comparative study in N Galicia (Spain). Energy 2015; 82: 138-146.

10. Mackay EB, Bahaj AS and Challenor PG. Uncertainty in wave energy resource assessment. Part 2: variability and predictability. Renew Energ 2010; 35(8): 1809-1819.

11. Parkinson SC, Dragoon K, Reikard G, et al. Integrating ocean wave energy at large-scales: a study of the US pacific Northwest. Renew Energ 2015; 76: 551-559.

12. Halamay DA, Brekken TK, Simmons A, et al. Reserve requirement impacts of large-scale integration of wind, solar, and ocean wave power generation. IEEE T Sustain Energ 2011; 2(3): 321-328.

13. Torsethaugen K. Bølgedata for vurdering av bølgekraft. Technical report STF60-A90120, 1990. Trondheim: SINTEF, 20 September.

14. Hasselmann K, Barnett T, Bouws E, et al. Measurements of wind-wave growth and swell decay during the Joint North Sea Wave Project (JONSWAP). Hamburg: Deutsches Hydrographisches Institut, 1973.

15. Bretschneider C. Wave variability and wave spectra for wind generated gravity waves. Technical report 118, August 1959. Washington, DC: Beach Erosion Board, US Army Corps of Engineers.

16. Folley M. Numerical modelling of wave energy converters. London: Academic Press, 2016.

17. Mackay EB, Bahaj AS and Challenor PG. Uncertainty in wave energy resource assessment. Part 1: historic data. Renew Energ 2010; 35(8): 1792-1808.

18. Penalba Retes M, Mérigaud A, Gilloteaux JC, et al. Nonlinear Froude-Krylov force modelling for two heaving wave energy point absorbers. In: Proceedings of the 11th European wave and tidal energy conference, Nantes, 6-11 September 2015.

19. Saulnier JB, Clément A, de O, António F, et al. Wave groupiness and spectral bandwidth as relevant parameters for the performance assessment of wave energy converters. Ocean Eng 2011; 38(1): 130-147. 
20. Kerbiriou MA, Prevosto M, Maisondieu C, et al. Influence of sea-states description on wave energy production assessment. In: Proceedings of the 7th European wave and tidal energy conference, Porto, 11-13 September 2007.

21. Spanos P, Di Paola M and Failla G. A Galerkin approach for power spectrum determination of nonlinear oscillators. Meccanica 2002; 37(1-2): 51-65.

22. Mérigaud A and Ringwood JV. A non-linear frequencydomain approach for numerical simulation of wave energy converters. IEEE T Sustain Energ. Epub ahead of print 16 June 2017. DOI: 10.1109/TSTE.2017.2716826.

23. Ochi MK. Ocean waves: the stochastic approach, vol. 6. Cambridge: Cambridge University Press, 2005.

24. Gallagher S, Tiron R, Whelan E, et al. The nearshore wind and wave energy potential of Ireland: a high resolution assessment of availability and accessibility. Renew Energ 2016; 88: 494-516.

25. Cummins W. The impulse response function and ship motion. Schiffstechnik 1962; 47(9): 101-109.

26. Bacelli G. Optimal control of wave energy converters. $\mathrm{PhD}$ Thesis, National University of Ireland, Maynooth, Maynooth, 2014.

27. Gilloteaux JC. Mouvements de grande amplitude d'un corps flottant en fluide parfait. Application à la récupération de l'énergie des vagues. $\mathrm{PhD}$ Thesis, Ecole Centrale de Nantes (ECN), Université de Nantes, Nantes, 2007.

28. Mérigaud A, Gilloteaux JC and Ringwood JV. A nonlinear extension for linear boundary element methods in wave energy device modelling. In: Proceedings of the ASME 2012 31st international conference on ocean, offshore and arctic engineering, Rio de Janeiro, Brazil, 1-6 July 2012, pp.615-621. New York: American Society of Mechanical Engineers.

29. Wheeler J. Methods for calculating forces produced by irregular waves. In: Proceedings of the offshore technology conference, Houston, TX, 18-21 May 1969.

30. Giorgi G and Ringwood JV. Computationally efficient nonlinear Froude-Krylov force calculations for heaving axisymmetric wave energy point absorbers. J Ocean Eng Mar Energ 2017; 3: 21-33.

31. Harris FJ. On the use of windows for harmonic analysis with the discrete Fourier transform. P IEEE 1978; 66(1): 51-83.

32. Mérigaud A and Ringwood JV. Free-surface time-series generation for wave energy applications. IEEE $J$ Oceanic Eng. Epub ahead of print 26 April 2017. DOI: 10.1109/ JOE.2017.2691199.

33. Barrett SN. Floating wave energy converters: wave measurement \& analysis techniques. PhD Thesis, University College Cork, Cork, 2015.

34. Saulnier JB, Ricci P, Clément A, et al. Mean power output estimation of WECs in simulated sea-states. In: Proceedings of the 8th European wave and tidal energy conference (EWTEC 2009), Uppsala, 7-10 September 2009, vol. 710 .

35. Tucker M, Challenor $\mathbf{P}$ and Carter D. Numerical simulation of a random sea: a common error and its effect upon wave group statistics. Appl Ocean Res 1984; 6(2): $118-122$.

36. Armesto JA, Guanche R, Del Jesus F, et al. Comparative analysis of the methods to compute the radiation term in Cummin's equation. J Ocean Eng Mar Energ 2015; 1(4): 377-393.

\section{Appendix I}

\section{Non-linear dynamical model for the spherical heaving point absorber}

The hydrodynamic model used for the sphere is a nonlinear extension of static and dynamic Froude-Krylov forces, inspired by the approach, first developed in Gilloteaux, ${ }^{27}$ later used in other studies such as Mérigaud et al. ${ }^{28}$ and Penalba Retes et al., ${ }^{18}$ and which consists of integrating static and incident pressure forces over the instantaneous wetted surface of the device.

The sphere is restricted in heave, along the axis $\vec{u}_{z}$ defined by $x=y=0$. As mentioned in WEC models, three main differences have to be stressed with respect to: ${ }^{27}$

- The dynamic pressure field is modified following a Wheeler stretching approach ${ }^{29}$ so that the total incident pressure, measured at the free surface, is exactly zero.

- The sphere is considered to be significantly smaller than the typical wave length so that, over the wetted surface of the sphere, the pressure field only depends on depth and time. Furthermore, the intersection between the device and the free surface is given in a simplified way, considering that, at a given instant $t$, the undisturbed free-surface height is uniformly equal to its value in $x=y=0$.

- Pressure integration over the instantaneous wetted surface is carried out analytically, like in Giorgi and Ringwood, ${ }^{30}$ instead of the panel method of Gilloteaux. $^{27}$

With the above assumptions, the approach of Gilloteau $x^{27}$ can be incorporated into the NLFD framework in a computationally efficient way, as detailed further in this section.

The static pressure force can be expressed as

$$
p_{s}(z)=-\rho g z
$$

For a monochromatic wave in infinite water depth, the dynamic incident pressure is written as

$$
p_{d y n}(z, t, \omega)=\Re\left\{\rho g A(\omega) \mathrm{e}^{j \omega t+k(\omega) z}\right\}
$$

where $k(\omega)=\omega^{2} / g$ is the wave number, and $A(\omega)$ denotes the complex wave amplitude.

With the Wheeler stretching approach, the dynamic pressure is reformulated, replacing $z$ with $\zeta=z-\eta$, the relative elevation with respect to the free surface

$$
p_{d y n}(\zeta, t, \omega)=\Re\left\{\rho g A(\omega) \mathrm{e}^{j \omega t+k(\omega) \zeta}\right\}
$$

The total incident dynamic pressure, due to the superposition of waves of different frequencies, is then

$$
p_{d y n}(\zeta, t)=\rho g \int_{0}^{\infty} \Re\left\{A(\omega) \mathrm{e}^{j \omega t}\right\} \mathrm{e}^{k(\omega) \zeta} d \omega
$$


It can be seen that, taken at the free surface $(\zeta=0)$, the static pressure equation (15) becomes $p_{s}=-\rho g \eta$, and the dynamic pressure equation (18) reduces to $p_{d y n}=\rho g \eta$ so that the total of the incident and static pressure is zero at the free surface.

The total force due to the static pressure over the immersed surface $\mathcal{S}_{\text {im }}$ of the body is

$$
\begin{aligned}
\vec{F}_{S}(t) & =-\int_{\mathrm{P} \in \mathcal{S}_{i m}}-\rho g z_{P} \vec{n} \mathrm{~d} \mathrm{~S} \\
& =\int_{\mathrm{P} \in \mathcal{S}_{i m}} \rho g \zeta_{P} \vec{n} \mathrm{~d} \mathrm{~S}+\int_{\mathrm{P} \in \mathcal{S}_{\text {im }}} \rho g \eta(t) \vec{n} \mathrm{dS} \\
& =\rho g \mathcal{V}_{i m}(t) \vec{u}_{z}-\rho g \eta(t) \mathcal{S}_{\text {int }}(t) \vec{u}_{z}
\end{aligned}
$$

where $\mathcal{V}_{\text {im }}$ denotes the immersed volume of the body, $\mathcal{S}_{\text {int }}$ denotes the area of the intersection between the body and the free surface (considered to be horizontal), and $\vec{u}_{z}$ is the unit vertical vector, pointing upwards.

For a sphere in heave with radius $R$ and density $\rho / 2$, denoting $\zeta_{G}$ the position of the sphere gravity centre relatively to the free surface, equation (19) can be analytically derived as

$$
\vec{F}_{s}\left(\zeta_{G}, t\right)=\rho g \pi\left[\frac{2}{3} R^{3}-R^{2} \zeta_{G}+\frac{1}{3} \zeta_{G}^{3}-\eta(t)\left(R^{2}-\zeta_{G}^{2}\right)\right] \vec{u}_{z}
$$

The total incident pressure force over the body can be written as

$$
\begin{aligned}
\vec{F}_{d y n} & =-\int_{\mathrm{P} \in \mathcal{S}_{i m}} p_{d y n}\left(z_{P}\right) \vec{n} \mathrm{~d} \mathrm{~S} \\
& =-\int_{\mathrm{P} \in \mathcal{S}_{\text {im }}} \rho g \int_{\omega=0}^{\infty} \Re\left\{A(\omega) \mathrm{e}^{j \omega t}\right\} \mathrm{e}^{k(\omega) \zeta} d \omega \vec{n} \mathrm{dS} \\
& =-\rho g \int_{\omega=0}^{\infty} \Re\left\{A(\omega) \mathrm{e}^{j \omega t}\right\}\left(\int_{\mathrm{P} \in \mathcal{S}_{i m}} \mathrm{e}^{k(\omega) \zeta} \vec{n} \mathrm{dS}\right) d \omega
\end{aligned}
$$

For a sphere, the integral over the immersed surface can be analytically derived, yielding

$$
\begin{aligned}
& \int_{\mathrm{P} \in \mathcal{S}_{i m}} \mathrm{e}^{k(\omega) \zeta} \vec{n} \mathrm{~d} \mathrm{~S}=2 \pi\left[-\frac{\zeta_{G}}{k(\omega)}-\frac{1}{k(\omega)^{2}}\right. \\
& \left.+\left(\frac{R}{k(\omega)}+\frac{1}{k(\omega)^{2}}\right) \mathrm{e}^{-k(\omega) R} \mathrm{e}^{k(\omega) \zeta_{G}}\right] \vec{u}_{z}
\end{aligned}
$$

Let us define, for $n \rho \digamma$

$$
\begin{aligned}
& I_{n}(t)=2 \pi \rho g \int_{0}^{\infty} \Re\left\{A(\omega) \mathrm{e}^{j \omega t}\right\} k(\omega)^{n} d \omega \\
& \text { and } \\
& J\left(\zeta_{G}, t\right)=2 \pi \rho g \int_{0}^{\infty} \Re\left\{A(\omega) \mathrm{e}^{j \omega t}\right\} \\
& \times\left(\frac{R}{k(\omega)}+\frac{1}{k(\omega)^{2}}\right) \mathrm{e}^{-k(\omega) R} \mathrm{e}^{k(\omega) \zeta_{G}} d \omega
\end{aligned}
$$

Thus, equation (21) can be expressed as

$$
\vec{F}_{d y n}\left(\zeta_{G}, t\right)=\left[I_{-1}(t) \zeta_{G}+I_{-2}(t)-J\left(\zeta_{G}, t\right)\right] \vec{u}_{z}
$$

At each iteration of the NLFD algorithm, as explained in NLFD simulation, equation (7), the

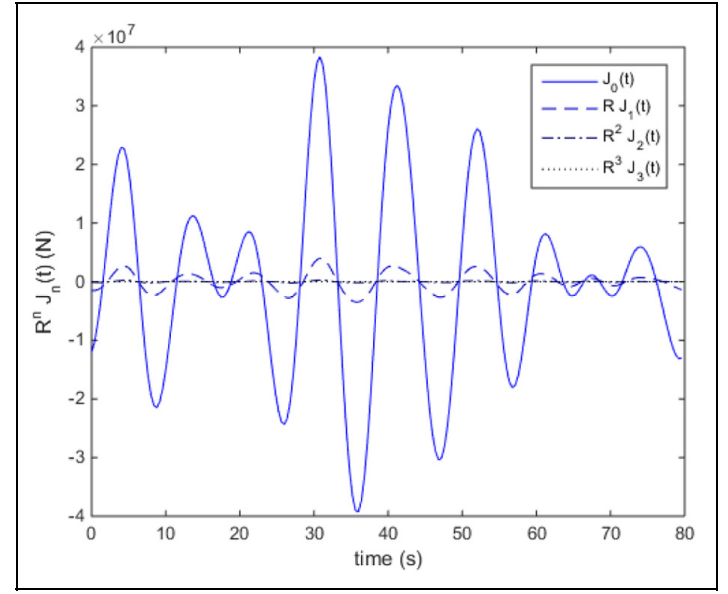

Figure 15. Terms $J_{n}(t) \zeta_{G}^{n}$ of expansion (27), taken at $\zeta_{G}=R$, for a typical JONSWAP spectrum $\left(H_{m_{0}}=2 \mathrm{~m}, T_{p}=10 \mathrm{~s}\right)$.

computation of non-linear forces and their derivatives necessitates an analytical expression of the forces as a function of the system variables in the time domain. However, using expression (25) into (7) would lead to a computationally expensive double integration over $\omega$ and $t$.

Thus, for a computationally efficient use within the NLFD algorithm, it is interesting to carry out a development of $J\left(\zeta_{G}, t\right)$, using a polynomial expansion of the exponential term, $\mathrm{e}^{\zeta_{G}}$. Defining

$$
\begin{aligned}
J_{n}(t)= & \frac{2 \pi \rho g}{n !} \int_{0}^{\infty} \Re\left\{A(\omega) \mathrm{e}^{j \omega t}\right\} \\
& \left(\frac{R}{k(\omega)}+\frac{1}{k(\omega)^{2}}\right) \mathrm{e}^{-k(\omega) R} k(\omega)^{n} d \omega
\end{aligned}
$$

Equation (25) can be formulated as

$$
\vec{F}_{d y n}\left(\zeta_{G}, t\right)=\left[I_{-1}(t) \zeta_{G}+I_{-2}(t)-\sum_{n=0}^{\infty} J_{n}(t) \zeta_{G}^{n}\right] \vec{u}_{z}
$$

so that the dynamic pressure force can be expanded to any arbitrary order $n_{\max }$ to reach a target degree of accuracy.

In this way, for a given periodic sea-state realisation (i.e. a set of complex amplitudes $A(\omega)$ ), the functions $I_{-1}(t), I_{-2}(t)$ and $J_{n}(t)$ are computed by means of FFTs prior to the start of the NLFD iterations. Then, at each iteration of the Newton method, the non-linear forces and their derivatives are computed in a simple polynomial form. Figure 15 shows the first three terms of the sum in equation (27), taken in the limit case $\zeta_{G}=R$, for a periodic wave signal derived from a typical JONSWAP spectrum $\left(H_{m_{0}}=2, T_{p}=10\right)$. As can be seen on Figure 15, the magnitude of the functions $J_{n}(t)$ decreases rapidly with the order $n$ so that, in practice, there is no noticeable difference between the results obtained with $n_{\max }=2$ or $n_{\max }=3$. In this article, $n_{\max }=3$ is chosen. 